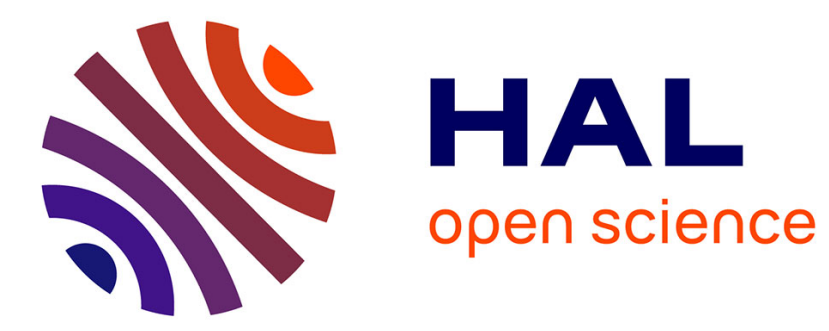

\title{
Local rank tests in a multivariate nonparametric relationship
}

N. Natércia Fortuna

\section{To cite this version:}

N. Natércia Fortuna. Local rank tests in a multivariate nonparametric relationship. Econometrics, 2007, 142 (1), pp.162. 10.1016/j.jeconom.2007.03.001 . hal-00501791

\section{HAL Id: hal-00501791 \\ https://hal.science/hal-00501791}

Submitted on 12 Jul 2010

HAL is a multi-disciplinary open access archive for the deposit and dissemination of scientific research documents, whether they are published or not. The documents may come from teaching and research institutions in France or abroad, or from public or private research centers.
L'archive ouverte pluridisciplinaire HAL, est destinée au dépôt et à la diffusion de documents scientifiques de niveau recherche, publiés ou non, émanant des établissements d'enseignement et de recherche français ou étrangers, des laboratoires publics ou privés. 


\section{Author's Accepted Manuscript}

Local rank tests in a multivariate nonparametric relationship

Natércia Fortuna

PII:

S0304-4076(07)00125-X

DOI: doi:10.1016/j.jeconom.2007.03.001

Reference: ECONOM 2954

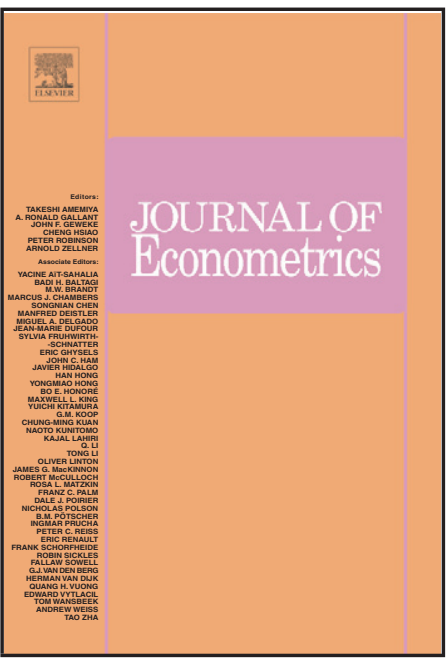

www.elsevier.com/locate/jeconom

To appear in: Journal of Econometrics

Received date: $\quad 8$ June 2004

Accepted date: $\quad 15$ March 2007

Cite this article as: Natércia Fortuna, Local rank tests in a multivariate nonparametric relationship, Journal of Econometrics (2007), doi:10.1016/j.jeconom.2007.03.001

This is a PDF file of an unedited manuscript that has been accepted for publication. As a service to our customers we are providing this early version of the manuscript. The manuscript will undergo copyediting, typesetting, and review of the resulting galley proof before it is published in its final citable form. Please note that during the production process errors may be discovered which could affect the content, and all legal disclaimers that apply to the journal pertain. 


\title{
Local rank tests in a multivariate nonparametric relationship
}

\author{
Natércia Fortuna* \\ CEMPRE $†$ Faculdade de Economia, Universidade do Porto
}

April 8, 2007

\begin{abstract}
Consider a multivariate nonparametric model where the unknown vector of functions depends on two sets of explanatory variables. For a fixed level of one set of explanatory variables, we provide consistent statistical tests, called local rank tests, to determine whether the multivariate relationship can be explained by a smaller number of functions. We also provide estimators for the smallest number of functions, called local rank, explaining the relationship. The local rank tests and the estimators of local rank are defined in terms of the eigenvalues of a kernel-based estimator of some matrix. The asymptotics of the eigenvalues is established by using the so-called Fujikoshi expansion along with some techniques of the theory of $U$-statistics. We present a simulation study which examines the small sample properties of local rank tests. We also apply the local rank tests and the local rank estimators to a demand system given by a newly constructed data set. This work can be viewed as a "local" extension of the tests for a number of factors in a nonparametric relationship introduced by Stephen Donald.
\end{abstract}

JEL classification: C12; C13; C14; D12

Keywords: Nonparametric relationship; Local rank; Kernel smoothing; Consistent tests; Demand systems

*Address: Faculdade de Economia do Porto, R. Dr. Roberto Frias, 4200-464 Porto, Portugal. Phone: (351) 225571 100. Fax: (351) 225505 050. E-mail: nfortuna@fep.up.pt. The author is grateful to Stephen G. Donald, Vladas Pipiras and the participants of the 2004 North American Summer Meeting of the Econometric Society in Brown University for their comments. The author would also like to thank an associate editor and three anonymous referees for many helpful suggestions. Thanks also to James Spencer and Pierre Bahizi.

${ }^{\dagger}$ CEMPRE - Centro de Estudos Macroeconómicos e Previsão - is supported by the Fundação para a Ciência e a Tecnologia, Portugal, through the Programa Operacional Ciência, Tecnologia e Inovação (POCTI) of the Quadro Comunitário de Apoio III, which is financed by FEDER and Portuguese funds. 


\section{Introduction}

This study was motivated by the theory of ranks of demand systems. Recall that a demand system is a functional relation $y=\left(y_{1}, \ldots, y_{J}\right)^{\prime}=f(x, z)=\left(f_{1}(x, z), \ldots, f_{J}(x, z)\right)^{\prime}$ where $y_{j}$, $j=1, \ldots, J$, is the proportion of the total expenditures for the $j$ th good, called a budget share for the $j$ th good, $x$ is total expenditures (income, in short) and $z=\left(z_{1}, \ldots, z_{J}\right)$ are prices of $J$ goods faced by a consumer. Introduced by Gorman (1981) and later developed by Lewbel (1991), the rank of a demand system can be either local or global. The local $\operatorname{rank} \operatorname{rk}\{f(\cdot, z)\}$ at a fixed value of $z$ is defined as the dimension of the function space spanned by the coordinate functions $f_{1}(x, z), \ldots, f_{J}(x, z)$ of $f(x, z)$ when $z$ is fixed. The global rank is the maximum of local ranks taken over all values of $z$. Ranks turn out to be of interest in Economic Theory where demand systems are derived through a utility maximization principle. For example, Gorman (1981) showed that commonly used exactly aggregable demand systems, when derived through a utility maximization principle, have always rank less than or equal to 3. Lewbel (1991) showed that ranks have important implications on functional structure and aggregation of demand systems. Some further theoretical studies related to ranks can be found in Lewbel (1989), Russell and Farris (1993), and Lewbel and Perraudin (1995).

Parallel to understanding its implications for Economic Theory, the rank of a demand system has been also studied from the point of view of a statistical estimation. A statistical model for a demand system is assumed to have a stochastic form $Y_{i}=f\left(X_{i}, Z_{i}\right)+\epsilon_{i}$, where $Y_{i}, X_{i}$ and $Z_{i}$ are the shares of goods, the income and the prices faced by the $i$ th consumer, and $\epsilon_{i}$ is the noise term. The rank of a stochastic system is defined in the same way as in the deterministic situation by using the coordinate functions of a vector $f(x, z)$. The function $f$ is assumed to have either a nonparametric or a (semi)parametric form. Under a semiparametric model, the rank of a demand system is typically expressed as the rank of some matrix. The rank of a matrix can be estimated by using one of the estimation procedures found in the literature, for example, the minimum- $\chi^{2}$ test of Cragg and Donald (1997), the LDU-based test of Gill and Lewbel (1992) (with a correction of Cragg and Donald (1996)) or the test based on SVD of Kleibergen and Paap (2006). Under a nonparametric model, the rank of a demand system is estimated by following the central work of Donald (1997). 
In most of the statistical work thus far, it has been assumed that prices are constant across consumers, that is, a model contains no variable $Z_{i}$. (In this case, there is no distinction between local and global rank tests.) One did so for simplicity and also because most data sets on consumer expenditure, in particular the well-known and commonly used Consumer Expenditures Survey (CEX, in short) data set of the United States, does not contain information on prices. The assumption of constant prices, however, is not realistic. For example, in the case of the U.S., the CEX data set covers households across all the U.S. and prices are clearly different in its various parts. The focus of this work is on extensions of the rank estimation problems to situations where variations in prices are taken into account. We will assume below a nonparametric form of a demand system and provide statistical tests to determine its local ranks. Estimation of local and global ranks in a particular (semi)parametric model can be found in Donald, Fortuna and Pipiras (2005). Estimation of global ranks in a non-parametric model, being less trivial, is left for the future work. Another significant part of this work consists of producing a data set which simultaneously contains information on expenditures and prices faced by a consumer in the U.S. We do so by matching the CEX data on expenditures of a consumer with the American Chamber of Commerce Researchers Association (ACCRA, in short) data of prices. A data set similar to ours was recently constructed and used by Nicol (2001) in the context of a statistical modeling of demand systems. Our work can also be viewed as a generalization of Donald (1997). Another related paper of interest is Kneip (1994). This article is an abridged version of Fortuna (2004) containing more details.

In view of our motivation described above, let $\left(X_{i}, Z_{i}\right) \in \mathbb{R}^{n} \times \mathbb{R}^{m}$ be independent variables and $Y_{i} \in \mathbb{R}^{G}$ be a response variable explained by $\left(X_{i}, Z_{i}\right)$. Suppose that the relationship between the variables $Y_{i}$ and $\left(X_{i}, Z_{i}\right)$ is given by the nonparametric model

$$
Y_{i}=F\left(X_{i}, Z_{i}\right)+U_{i}, \quad i=1, \ldots, N
$$

where $N$ is the number of observations, $F(x, z)=\left(F_{1}(x, z), \ldots, F_{G}(x, z)\right)^{\prime}$ is an unknown $G \times 1$ vector of functions of $x$ and $z$, and $U_{i}$ is a $G \times 1$ noise vector with the covariance matrix

$$
\Sigma=E U_{i} U_{i}^{\prime}
$$

One of the key assumptions of this work is the non-singularity (invertibility) of the matrix $\Sigma$. 
Additional assumptions on the variables $X_{i}, Z_{i}$ and $U_{i}$, and on the function $F$ are stated in Section 3. To state the problems considered in this paper, we need the following definition generalizing the notion of a local $\operatorname{rank} \operatorname{rk}\{F(\cdot, z)\}$ introduced earlier.

Definition 1.1 Define the local rank of a $G \times 1$ vector $F(x, z)$ at $z$ (and related to a $d_{1} \times 1$ subvector $x^{1}$ of $x$ ), denoted by

$$
\operatorname{rk}\left\{F(\cdot, z) ; x^{1}\right\}
$$

as the smallest integer $L$ such that, for a $d_{1} \times 1$ subvector $x^{1}$ of $x$, a $G \times d_{1}$ matrix $c(z)$, a $G \times L$ matrix $A(z)$ and a $L \times 1$ vector $H(x, z)$, we have

$$
F(x, z)=c(z) x^{1}+A(z) H(x, z) .
$$

By $\operatorname{rk}\{F(\cdot, z) ; 0\}$, also denoted by $\operatorname{rk}\{F(\cdot, z)\}$, we shall mean the smallest $L$ such that the decomposition (1.3) holds without the term $c(z) x^{1}$.

Observe that the definition of the local $\operatorname{rank} \operatorname{rk}\{F(\cdot, z) ; 0\}=\operatorname{rk}\{F(\cdot, z)\}$ is equivalent to that given in the beginning of the section. Why then introduce the notion of a more general local rank? The answer goes back to the assumption of the non-singularity of the covariance matrix $\Sigma=E U_{i} U_{i}^{\prime}$ which we will use. If $Y_{i}=f\left(X_{i}, Z_{i}\right)+\epsilon_{i}$ is a nonparametric model of a demand system, then the sum of the budget shares $Y_{i j}, j=1, \ldots, J$, in $Y_{i}=\left(Y_{i 1}, \ldots, Y_{i J}\right)$ is always equal to 1 . This implies that the covariance matrix of $\epsilon_{i}$ is singular and hence that the results of the paper do not, in principle, apply because they rely on non-singular covariance matrices. The way out is to observe that, because of the sum to 1 condition, $\operatorname{rk}\{f(\cdot, z)\}=\operatorname{rk}\{F(\cdot, z) ; 1\}+1$, where $F(\cdot, z)$ is a vector $f(\cdot, z)$ without any one of its coordinate functions and $\operatorname{rk}\{F(\cdot, z) ; 1\}$ is defined by Definition 1.1 with $d_{1}=1$ and $x^{1}=1$ (see Lemma B.10 in Appendix B). Then, to estimate the local rank of a demand system, drop one share of goods from the analysis, allowing to assume non-singular covariance matrix of disturbances, estimate $\operatorname{rk}\{F(\cdot, z) ; 1\}$ and add 1 to the result. From this perspective, why then consider the local $\operatorname{ranks} \operatorname{rk}\{F(\cdot, z)\}$ and $\operatorname{rk}\left\{F(\cdot, z) ; x^{1}\right\}$ with $x^{1} \neq 1$ ? We include these ranks in Definition 1.1 because of potential applications to problems other than the rank of a demand system and also because our proofs in the case of $\operatorname{rk}\{F(\cdot, z) ; 1\}$ and in the general case of $\operatorname{rk}\left\{F(\cdot, z) ; x^{1}\right\}$ are not very different. In 
addition, Definition 1.1 follows the framework of Donald (1997) where our general rank with no $z$ is also implicitly defined.

In this work, we focus on and address the following problems related to local rank $\operatorname{rk}\left\{F(\cdot, z) ; x^{1}\right\}$.

Basic problems. For fixed $z$ and $L$, provide statistical tests for the hypothesis testing problem of $H_{0}: \operatorname{rk}\left\{F(\cdot, z) ; x^{1}\right\} \leq L$ against the alternative $H_{1}: \operatorname{rk}\left\{F(\cdot, z) ; x^{1}\right\}>L$. For a fixed $z$, provide an estimator for the local $\operatorname{rank} \operatorname{rk}\left\{F(\cdot, z) ; x^{1}\right\}$.

Since $z$ is fixed, we will refer to the above statistical tests as local rank tests or rank tests local at z. The basic idea behind these local rank tests, explained in greater detail in Section 2 below, is to relate the local $\operatorname{rank} \operatorname{rk}\left\{F(\cdot, z) ; x^{1}\right\}$ to the number of zero eigenvalues of some matrix. Then, by testing for the number of zero eigenvalues of this matrix, one can make an inference about the local $\operatorname{rank} \operatorname{rk}\left\{F(\cdot, z) ; x^{1}\right\}$. The difficult parts of this plan are to find the right matrix, to obtain its estimator and, finally, to prove the asymptotics of the eigenvalues of the estimator which would allow to distinguish between the two hypothesis. The goal of the paper is to show how these difficulties can be overcome.

The rest of the paper is structured as follows. In Section 2, we explain the basic idea behind the local rank tests and also introduce the related estimators. In Section 3, we state our assumptions. In Section 4, we establish the asymptotic properties of the estimated eigenvalues of some matrix and, based on these properties, we formulate the local rank tests and discuss estimation of the local rank itself. Simulation results and applications to demand systems can be found in Sections 5 and 6, respectively. Finally, in Section 7, we draw some conclusions. The proofs of all the results can be found in Appendices A and B. Appendix C contains a result on asymptotics of a second order $U$-statistics.

\section{Preliminaries}

The basic idea behind local rank tests for (NP) model lies in the following lemma. The notation $\operatorname{rk}\{A\}$ stands for the rank of a matrix $A$. 
Lemma 2.1 For fixed $z$ and $L$, we have $\operatorname{rk}\left\{F(\cdot, z) ; x^{1}\right\} \leq L$ if and only if the matrix

$$
\Gamma_{w, z}=E \gamma\left(X_{i}, z\right) \widetilde{F}\left(X_{i}, z\right) \widetilde{F}\left(X_{i}, z\right)^{\prime}
$$

where $\gamma(x, z)>0$ is any real-valued function and

$$
\widetilde{F}(x, z)=F(x, z)-E \beta\left(X_{i}, z\right) F\left(X_{i}, z\right) X_{i}^{1^{\prime}}\left(E \beta\left(X_{i}, z\right) X_{i}^{1} X_{i}^{1^{\prime}}\right)^{-1} x^{1}
$$

with any real-valued function $\beta(x, z) \neq 0$, has $G-L$ zero eigenvalues, or if and only if the matrix $\Gamma_{w, z} \Sigma^{-1}$ has $G-L$ zero eigenvalues, where $\Sigma$ is defined in (1.1). This is also equivalent to

$$
\operatorname{rk}\left\{F(\cdot, z) ; x^{1}\right\}=\operatorname{rk}\left\{\Gamma_{w, z}\right\}=\operatorname{rk}\left\{\Gamma_{w, z} \Sigma^{-1}\right\}
$$

Local rank tests for (NP) model will then be based upon the smallest $G-L$ eigenvalues of an estimator of the matrix $\Gamma_{w, z} \Sigma^{-1}$. As can be seen from the proof of Theorem 4.1 below, the matrix $\Sigma^{-1}$ plays the role of a normalization in order to obtain standardized limit laws. The weights $\gamma(x, z)$ and $\beta(x, z)$ are taken for convenience to allow for easier manipulations. We will take

$$
\gamma(x, z)=\frac{p(x, z)^{2}}{\widetilde{p}(x)}, \quad \beta(x, z)=\frac{p(x, z)}{\widetilde{p}(x)}
$$

where $p(x, z)$ and $\widetilde{p}(x)$ are the densities of the vector $(X, Z)$ and the variable $X$, respectively.

We will define the estimators for the matrices $\Gamma_{w, z}$ and $\Sigma$ by using kernel functions. Set $x^{b}=x_{1}^{b_{1}} \ldots x_{m}^{b_{m}}$ and $|b|=b_{1}+\ldots+b_{m}$ for $b=\left(b_{1}, \ldots, b_{m}\right) \in(\mathbb{N} \cup\{0\})^{m}$ and $x=\left(x_{1}, \ldots, x_{m}\right) \in$ $\mathbb{R}^{m}$. A function $K: \mathbb{R}^{m} \rightarrow \mathbb{R}$ is a kernel of order $r \in \mathbb{N}$ on $\mathbb{R}^{m}$ if it has a compact support, is bounded and satisfies the following conditions: $(i) \int_{\mathbb{R}^{m}} K(x) d x=1$ and $(i i) \int_{\mathbb{R}^{m}} x^{b} K(x) d x=$ 0 for any $b \in(\mathbb{N} \cup\{0\})^{m}$ such that $1 \leq|b|<r$. Kernel functions are useful because of their localization property (see Proposition B.1 in Appendix B) which involves a scaled kernel function $K_{h}(\cdot)=h^{-m} K\left(h^{-1} \cdot\right)$, where $h>0$ is called a bandwidth.

Definition 2.1 Define the estimator of the matrix $\Gamma_{w, z}$ in (2.1) with (2.2) and (2.4) as

$$
\widehat{\Gamma}_{w, z}=\frac{1}{N(N-1)} \sum_{i \neq j}^{N}\left(Y_{i}-\widehat{\Pi}_{1}(z)^{\prime} X_{i}^{1}\right)\left(Y_{j}-\widehat{\Pi}_{1}(z)^{\prime} X_{j}^{1}\right)^{\prime} \widetilde{K}_{h}\left(X_{i}-X_{j}\right) K_{h}\left(z-Z_{i}\right) K_{h}\left(z-Z_{j}\right)
$$

where $\widehat{\Pi}_{1}(z)^{\prime}$ is given by

$$
\widehat{\Pi}_{1}(z)^{\prime}=\frac{1}{N} \sum_{i=1}^{N} Y_{i} X_{i}^{1^{\prime}} K_{h}\left(z-Z_{i}\right)\left(\frac{1}{N} \sum_{i=1}^{N} X_{i}^{1} X_{i}^{1^{\prime}} K_{h}\left(z-Z_{i}\right)\right)^{-1} .
$$


Remark 2.1 We expect the estimator

$$
\widehat{\Gamma}_{w, z}=\frac{1}{N(N-1)} \sum_{i \neq j}^{N} Y_{i} Y_{j}^{\prime} \widetilde{K}_{h}\left(X_{i}-X_{j}\right) K_{h}\left(z-Z_{i}\right) K_{h}\left(z-Z_{j}\right)
$$

to be used for tests of local $\operatorname{rank} \operatorname{rk}\{F(\cdot, z)\}=\operatorname{rk}\{F(\cdot, z) ; 0\}$. The correction $\widehat{\Pi}_{1}(z)^{\prime} X_{i}^{1}$ appears in (2.5) to account for the term $c(z) x^{1}$ in the general definition of a local $\operatorname{rank} \operatorname{rk}\left\{F(\cdot, z) ; x^{1}\right\}$.

We shall also use the standard estimator of the covariance matrix $\Sigma$ in (1.1) given by

$$
\widehat{\Sigma}=\frac{1}{N} \sum_{i=1}^{N}\left(Y_{i}-\widehat{F}\left(X_{i}, Z_{i}\right)\right)\left(Y_{i}-\widehat{F}\left(X_{i}, Z_{i}\right)\right)^{\prime},
$$

where

$$
\widehat{F}(x, z)=\frac{1}{N} \sum_{i=1}^{N} Y_{i} \widetilde{K}_{h}\left(x-X_{i}\right) K_{h}\left(z-Z_{i}\right) \widehat{p}(x, z)^{-1}
$$

and

$$
\widehat{p}(x, z)=\frac{1}{N} \sum_{i=1}^{N} \widetilde{K}_{h}\left(x-X_{i}\right) K_{h}\left(z-Z_{i}\right)
$$

\section{Assumptions}

In this section, we list and briefly discuss the assumptions which will be used for local rank tests.

Assumption 1: Suppose that $\left(X_{i}, Z_{i}\right) \in \mathbb{R}^{n} \times \mathbb{R}^{m}, i=1, \ldots, N$, are i.i.d. random vectors such that the support of $\left(X_{i}, Z_{i}\right)$, denoted by $\mathcal{H}_{x} \times \mathcal{H}_{z}$, is the Cartesian product of compact intervals and $\left(X_{i}, Z_{i}\right)$ are continuously distributed with a density $p(x, z)$ which is bounded below by a constant and has an extension to $\mathbb{R}^{n} \times \mathbb{R}^{m}$ with $s \geq r$ continuous bounded derivatives.

Assumption 2: Suppose that $U_{i}, i=1, \ldots, N$, are i.i.d. random vectors, independent of the sequence $\left(X_{i}, Z_{i}\right)$ and such that $E U_{i}=0$ and $E U_{i} U_{i}^{\prime}=\Sigma$, where $\Sigma$ is a positive definite matrix. Assume also that $E U_{j}^{4}<\infty$.

Assumption 3: The function $F: \mathcal{H}_{x} \times \mathcal{H}_{z} \rightarrow \mathbb{R}^{G}$ is such that each of its component functions has an extension to $\mathbb{R}^{n} \times \mathbb{R}^{m}$ with $s \geq r$ continuous bounded derivatives.

Assumption 4: The matrix

$$
Q_{1}(z)=E \frac{p\left(X_{1}, z\right)}{\widetilde{p}\left(X_{1}\right)} X_{1}^{1} X_{1}^{1^{\prime}}=\int_{\mathbb{R}^{n}} x_{1}^{1} x_{1}^{1^{\prime}} p\left(x_{1}, z\right) d x_{1},
$$


is positive definite (invertible), where $\widetilde{p}(x)$ is the density function of $X_{i}$.

Assumption 5: The functions $\widetilde{K}$ and $K$ are symmetric kernels on $\mathbb{R}^{n}$ and $\mathbb{R}^{m}$, respectively, of order $r$.

Assumptions 1-4 are in the spirit of those used by Donald (1997). Assumptions 1 and 3 require smoothness of the true nonparametric regression function and the density of $\left(X_{i}, Z_{i}\right)$. Assumption 4 requires that the limit of the inverse matrix in (2.6) is well-defined. Some of the above assumptions can be slightly weakened, for example, by replacing the independence condition on $U_{i}$ in Assumption 2 by suitable behavior of $U_{i}$ given $X_{i}$ and $Z_{i}$. We shall, however, not strive here for utmost generality and leave the assumptions which are easier to work with.

\section{Local rank tests and estimation of local rank}

The following result is key to local rank tests for (NP) model. Let $\widehat{\lambda}_{1}(z) \leq \ldots \leq \widehat{\lambda}_{G}(z)$ be the eigenvalues of the matrix $\widehat{\Gamma}_{w, z} \widehat{\Sigma}^{-1}$. Set

$$
V(z)=\left(2\|\widetilde{K}\|_{2}^{2}\|K\|_{2}^{4} E \frac{p\left(X_{i}, z\right)^{2}}{\widetilde{p}\left(X_{i}\right)}\right)^{-1 / 2}
$$

and let

$$
\widehat{V}(z)=\left(2\|\widetilde{K}\|_{2}^{2}\|K\|_{2}^{4} N^{-1} \sum_{i=1}^{N} \widehat{p}\left(X_{i}, Z_{i}\right) K_{h}\left(z-Z_{i}\right)\right)^{-1 / 2},
$$

where $\widehat{p}(x, z)$ is given in $(2.10)$. By Lemma B.8 below, under suitable conditions, $\widehat{V}(z)$ is a consistent estimator of $V(z)$. Let also $\mathcal{Z}_{k}$ be a symmetric $k \times k$ matrix having independent zero mean normal (Gaussian) entries with variance 1 in the diagonal and variance 1/2 off the diagonal, and $\lambda_{1}\left(\mathcal{Z}_{k}\right) \leq \ldots \leq \lambda_{k}\left(\mathcal{Z}_{k}\right)$ be the eigenvalues of $\mathcal{Z}_{k}$ in the increasing order.

Theorem 4.1 Suppose that Assumptions 1-5 of Section 3 hold, and that

$$
N h^{m+3 n / 2} \rightarrow \infty \quad \text { and } \quad N h^{m+n / 2+2 r} \rightarrow 0
$$

Set $L(z)=\operatorname{rk}\left\{F(\cdot, z) ; x^{1}\right\}$. Then, for $j=1, \ldots, G-L(z)$,

$$
\widehat{V}(z) N h^{m+n / 2} \widehat{\lambda}_{j}(z) \stackrel{d}{\rightarrow} \lambda_{j}\left(\mathcal{Z}_{G-L(z)}\right),
$$

and, for $j=G-L(z)+1, \ldots, G$,

$$
\widehat{V}(z) N h^{m+n / 2} \widehat{\lambda}_{j}(z) \stackrel{p}{\rightarrow}+\infty
$$


We now state an immediate corollary of Theorem 4.1 which can be used in local rank tests for (NP) model, that is, to test $H_{0}: \operatorname{rk}\left\{F(\cdot, z) ; x^{1}\right\} \leq L$ against $H_{1}: \operatorname{rk}\left\{F(\cdot, z) ; x^{1}\right\}>L$, in a standard way. Let

$$
\widehat{T}_{1}(L, z)=\frac{\widehat{V}(z) N h^{m+n / 2}}{\sqrt{G-L}} \sum_{j=1}^{G-L} \widehat{\lambda}_{j}(z)
$$

and

$$
\widehat{T}_{2}(L, z)=\widehat{V}(z)^{2} N^{2} h^{2 m+n} \sum_{j=1}^{G-L}\left(\widehat{\lambda}_{j}(z)\right)^{2} .
$$

Recall that a stochastic dominance $\xi \leq_{d} \eta$ means that $P(\xi>x) \leq P(\eta>x)$ for all $x \in \mathbb{R}$. The notation $\chi^{2}(k)$ below stands for a $\chi^{2}$-distribution with $k$ degrees of freedom.

Theorem 4.2 Under the assumptions of Theorem 4.1, we have that, under the hypothesis $H_{0}: \operatorname{rk}\left\{F(\cdot, z) ; x^{1}\right\} \leq L$,

$$
\widehat{T}_{1}(L, z) \stackrel{d}{\rightarrow} \frac{1}{\sqrt{G-L}} \sum_{j=1}^{G-L} \lambda_{j}\left(\mathcal{Z}_{G-L(z)}\right) \stackrel{d}{\leq} \mathcal{N}(0,1)
$$

and

$$
\widehat{T}_{2}(L, z) \stackrel{d}{\rightarrow} \sum_{j=1}^{G-L}\left(\lambda_{j}\left(\mathcal{Z}_{G-L(z)}\right)\right)^{2} \stackrel{d}{\leq} \chi^{2}((G-L)(G-L+1) / 2),
$$

where the stochastic dominance $\leq_{d}$ in (4.8) and (4.9) is, in fact, $=_{d}$ for $L=\operatorname{rk}\left\{F(\cdot, z) ; x^{1}\right\}$, and, under the hypothesis $H_{1}: \operatorname{rk}\left\{F(\cdot, z) ; x^{1}\right\}>L, \widehat{T}_{i}(L, z) \rightarrow_{p}+\infty, i=1,2$.

Remark 4.1 Relation (2.3) indicates that local rank tests can be viewed as tests for the rank of the matrix $\Gamma_{w, z}$. We cannot readily apply the rank tests available in the literature (see Section 1) because they are formulated for matrices with no restrictions. The matrices $\Gamma_{w, z}$ and $\widehat{\Gamma}_{w, z}$, on the other hand, are necessarily symmetric. Symmetry imposes restrictions on matrices involved and consequently alters rank test results. We plan to explore these issues under general framework in a future work.

Remark 4.2 The bandwidths $h$ corresponding to $X_{i}$ and $Z_{i}$ in (2.5) play somewhat different roles. Hence, particularly in practice, one may want to consider the test statistic

$$
\widehat{\Gamma}_{w, z}=\frac{1}{N(N-1)} \sum_{i \neq j}^{N}\left(Y_{i}-\widehat{\Pi}_{1}(z)^{\prime} X_{i}^{1}\right)\left(Y_{j}-\widehat{\Pi}_{1}(z)^{\prime} X_{j}^{1}\right)^{\prime} \widetilde{K}_{h_{x}}\left(X_{i}-X_{j}\right) K_{h_{z}}\left(z-Z_{i}\right) K_{h_{z}}\left(z-Z_{j}\right),
$$


where $h_{x}, h_{z}>0$. One may show that, under suitable conditions and using the normalization $N h_{z}^{m} h_{x}^{n / 2}$, the eigenvalues $\widehat{\lambda}_{j}(z)$ of the matrix $\widehat{\Gamma}_{w, z} \widehat{\Sigma}^{-1}$ (where $\widehat{\Gamma}_{w, z}$ is defined in (4.10)) satisfy the limit results analogous to those in Theorems 4.1 and 4.2. In our simulation study and applications below, we will consider the test statistic (4.10) and use the normalization $N h_{z}^{m} h_{x}^{n / 2}$.

Local rank can be estimated through a commonly used sequential testing. In the case of the tests statistic $\widehat{T}_{1}(L, z)$, consider

$$
\widehat{L}_{1}(z)=\min \left\{L: \widehat{T}_{1}(L, z)<\mathcal{N}_{\alpha}(0,1)\right\}
$$

where $\xi=\mathcal{N}_{\alpha}(0,1)$ satisfies $P(\mathcal{N}(0,1)>\xi)=\alpha$, that is, the first $L$ at which the hypothesis $H_{0}: \operatorname{rk}\left\{F(\cdot, z) ; x^{1}\right\} \leq L$ is not rejected at a level of significance $\alpha$. To make $\widehat{L}_{1}(z)$ consistent, it is necessary to have $\alpha=\alpha(N, h) \rightarrow 0$ at a suitable rate as $N \rightarrow \infty$ and $h \rightarrow 0$. When using the test statistic $\widehat{T}_{2}(L, z)$, we need to consider

$$
\widehat{L}_{2}(z)=\min \left\{L: \widehat{T}_{2}(L, z)<\chi_{\alpha(N, h)}^{2}((G-L)(G-L+1) / 2)\right\},
$$

where $\xi=\chi_{\alpha(N, h)}^{2}((G-L)(G-L+1) / 2)$ is such that $P\left(\chi^{2}((G-L)(G-L+1) / 2)>\xi\right)=\alpha(N, h)$.

Theorem 4.3 With the above notation and under the assumptions of Theorem 4.1, we have $\widehat{L}_{i}(z) \rightarrow{ }_{p} \operatorname{rk}\left\{F(\cdot, z) ; x^{1}\right\}, i=1,2$, as long as $\alpha(N, h) \rightarrow 0$ and $(-\ln \alpha(N, h))^{1 / 2} / N h^{m+n / 2} \rightarrow 0$, when $i=1$, and $\alpha(N, h) \rightarrow 0$ and $-\ln \alpha(N, h) / N^{2} h^{2 m+n} \rightarrow 0$, when $i=2$.

\section{Simulation study}

In this section, we use Monte Carlo simulations to examine size and power properties of local rank tests and properties of local rank estimators obtained through sequential testing. We also compare local rank estimation to other rank estimation procedures, e.g. the situation when the variable $z$ (being part of the nonparametric model) is ignored. For simplicity, we focus henceforth only on local $\operatorname{ranks} \operatorname{rk}\{F(\cdot, z)\}=\operatorname{rk}\{F(\cdot, z) ; 0\}$ (see Definition 1.1).

The experimental setup is as follows. We consider $Y_{i}=\delta F\left(X_{i}, Z_{i}\right)+U_{i}, i=1, \ldots, N$, where

$$
F(x, z)=\left(\begin{array}{c}
\frac{5+z}{1+x^{4}} \sin \left(2.5+x^{4}\right) \\
\frac{1}{3}(2-z)\left(z+\frac{1}{2}\right) \arctan (5(x-1-\gamma z)) \\
z\left(z+\frac{1}{2}\right)\left(1+z^{2}\right)
\end{array}\right) .
$$


We suppose that $\left(X_{i}, Z_{i}\right), i=1, \ldots, N$, are independent identically distributed $\mathbb{R}^{2}$ random vectors: $X_{i}$ and $Z_{i}$ are distributed uniformly on $[0,2]$ and $[-1,1]$, respectively, and $X_{i}$ and $Z_{i}$ are independent. We consider two sample sizes $N=750$ and $N=1500$, and two signal-tonoise $\operatorname{ratios} \delta=1$ and $\delta=1 / 2$. The noise variables $U_{i}$ are normally distributed as $\mathcal{N}\left(0, I_{3}\right)$, where $I_{3}$ is a $3 \times 3$ identity matrix. We suppose $\gamma=1$ though we shall also discuss briefly the case $\gamma=2$. We shall estimate local $\operatorname{ranks} \operatorname{rk}\{F(\cdot, z)\}$ at $z=1 / 2, z=0$ and $z=-1 / 2$ corresponding to $\operatorname{rk}\{F(\cdot, 1 / 2)\}=3, \operatorname{rk}\{F(\cdot, 0)\}=2$ and $\operatorname{rk}\{F(\cdot,-1 / 2)\}=1$, respectively. Size and power computations will be based on local rank tests for these values of $z$. The number of replications in Monte Carlo simulations is 1000 throughout. For kernel smoothing, we use a popular Epanechnikov kernel. We shall also focus in this section on local rank tests based on the test statistic $\widehat{T}_{1}(L, z)$.

Table 1 presents size computations as actual rejection frequencies of local rank tests, based on the test statistic $\widehat{T}_{1}(L, z)$. The interval $[0.1,0.5]$ corresponding to the smallest and largest values of $h_{x}$ and $h_{z}$, contains most of the bandwidths which where obtained through the generalized and the usual cross validations in a number of Monte Carlo replications. For example, when $N=750$ and $\delta=1$, the generalized cross validation chose the values $\left(h_{x}, h_{z}\right)=(0.2,0.4)$ and $(0.2,0.3)$ in 30 Monte Carlo replications. To compare a nonparametric approach to a parametric one, we also present in Table 1 sizes computed from a fixed parametric model. More precisely, we fit to the data a semiparametric model $Y_{i}=A\left(Z_{i}\right) H\left(X_{i}\right)+\epsilon_{i}$, where

$$
H(x)=\left(\begin{array}{c}
1 \\
x \\
(x-1)^{2}
\end{array}\right)
$$

and $A(z)$ is unknown. The local $\operatorname{rank} \operatorname{rk}\{F(\cdot, z)\}$ of the system $F(x, z)=A(z) H(x)$ can be shown to be the rank of the matrix $A(z)$. We therefore estimate $A(z)$ by using kernel smoothing and then test for its rank by using a minimum- $\chi^{2}$ test for the rank of a matrix (Cragg and Donald (1997)). For more information on rank estimation in this model, see Donald et al. (2005).

A few observations can be drawn from Table 1. The results indicate that local rank tests are likely to be undersized. Undersizing is most pronounced for local rank $2(z=0)$. Moreover, observe that the size appears to increase as $h_{z}$ becomes larger, and it appears to get optimal as $h_{x}$ decreases. The latter observation suggests that we should use $h_{x}$ corresponding 
to undersmoothing. The same finding was also reported by Donald (1997) in the context of nonparametric rank testing without $z$. What $h_{z}$ should be used is less clear, especially for rank 1. For rank 2 , on the other hand, a larger $h_{z}$ appears to be optimal. Then, we should perhaps use $h_{z}$ corresponding to oversmoothing. Comparing nonparametric and parametric approaches, we see that local rank tests have significantly greater sizes for the parametric model. This discrepancy possibly results from the fact that the first function in (5.1), for example, is not easily approximated by a second order polynomial.

Power computations for local rank tests are presented in Table 2. These are size adjusted powers where the critical values used for local rank tests of $H_{0}: \operatorname{rk}\{F(\cdot, z)\} \leq L$ are taken to make sizes equal to 5 percent in Table 1 . The results of Table 2 suggest that power of local rank tests increases as $h_{x}$ and $h_{z}$ become larger. Observe, however, that the loss in power is not very significant as long as $h_{z}$ is not too small. When the variable $z$ is ignored, Donald (1997) has also observed that there is little loss in power when $h_{x}$ decreases. Together with the results of Table 1 , this observation suggests that using smaller $h_{x}$ corresponding to undersmoothing and larger $h_{z}$ corresponding to oversmoothing, may be more optimal for local rank tests. Observe also that powers obtained by fitting the semiparametric model are comparable to those of a nonparametric approach. This finding surprised us since we expected a greater loss in power for the parametric approach, similarly to the results found in Donald (1997). In our understanding, this difference from Donald (1997) is just the result of our model choice (5.1).

In Table 3, we also provide empirical distributions of local rank estimator obtained through sequential testing at a constant significance level $\alpha=0.05$, for local $\operatorname{ranks} \operatorname{rk}\{F(\cdot,-1 / 2)\}$ (true $\left.\operatorname{rank} L_{0}=1\right), \operatorname{rk}\{F(\cdot, 0)\}$ (true $\operatorname{rank} L_{0}=2$ ) and $\operatorname{rk}\{F(\cdot, 1 / 2)\}$ (true rank $L_{0}=3$ ), when $N=750$. It is quite remarkable that local rank tests do fairly well when $N=750, \delta=1$. (The results for $N=1500$ not reported here, are even better.) The results appear poor for $N=750, \delta=1 / 2$ but we should not expect them better because there is just too much noise in the data.

Finally, in Table 4, we present empirical distribution of estimated $\operatorname{rank} \widehat{L}$ when the variable $z$ is ignored altogether. In other words, though still generating the variables $\left(Y_{i}, X_{i}, Z_{i}\right)$ as above, we now focus only on the data $\left(Y_{i}, X_{i}\right), i=1, \ldots, N=750$, and estimate its rank either by Donald's (1997) nonparametric approach or by a minimum- $\chi^{2}$ test as the rank of a regression 
coefficient matrix after fitting a quadratic parametric model. It is interesting to compare the results of Table 4 to those of Table 3. For example, observe that the results of Table 4 for $\widehat{L}=3$ are a little smaller but still comparable to those in Table 3 for the true rank $L_{0}=3, \widehat{L}=3$ (with $h_{z}=0.3$ or 0.5$)$. Since $L_{0}=3=\operatorname{rk}\{F(\cdot, 1 / 2)\}=\max _{z} \operatorname{rk}\{F(\cdot, 1 / 2)\}$ for the function system (5.1), one might think that the rank estimated ignoring the value $z$, provides a good estimator for the global rank $\max _{z} \operatorname{rk}\{F(\cdot, z)\}$. Our guess is that, depending on the model, this will not always be true. A simple example is the function system (5.1) with $\gamma=2$. Since the argument $5(x-1-2 z)$ of the second function in (5.1) can take now a much larger range of values, the second coordinate of the data $Y_{i}$ will appear much more like a white noise as a function of $X_{i}$ $\left(Z_{i}\right.$ being ignored). Hence, the rank estimated ignoring $z$ will not concentrate at 3 as in Table 4 but at the value 2 . We have studied the data of this example through simulations. The obtained results confirmed our guess.

We have also performed all of the above simulations with the alternative test statistic $\widehat{T}_{2}(L, z)$. The results not reported here, suggest that the statistic $\widehat{T}_{2}(L, z)$ slightly overestimates (respectively, underestimates) the local rank as compared to $\widehat{T}_{1}(L, z)$ when the true rank is not full, that is, the true rank $L_{0}=1$ or $L_{0}=2$ in Table 3 (respectively, the true rank is full, that is the true rank $L_{0}=3$ in the table). This observation translates into the fact that the tests based on $\widehat{T}_{2}(L, z)$ have better size properties than those based on $\widehat{T}_{1}(L, z)$. The power of tests based on $\widehat{T}_{2}(L, z)$ is typically worse but still comparable to that based on $\widehat{T}_{1}(L, z)$ in most cases of practical interest. (There is a significant difference in power for $L_{0}=3, L=2$ and $\delta=1$ when $h_{x}$ or $h_{z}$ is the smallest, and for $L_{0}=3, L=2$ when the signal-to-noise ratio $\delta=1 / 2$ is the smallest.) Since the tests based on $\widehat{T}_{2}(L, z)$ have better size properties, this suggests that using the statistic $\widehat{T}_{2}(L, z)$ may often be more reliable than using the statistic $\widehat{T}_{1}(L, z)$.

\section{Application to demand system}

In this section, we estimate local rank in a demand system. The data set which we use contains information on expenditures and prices faced by a number of consumers across the U.S. Expenditures are taken from the U.S. CEX micro data of the first quarter of $2000^{1}$. More specifically,

\footnotetext{
${ }^{1}$ U.S. Dep. of Labor, Bureau of Labor Statistics. Consumer Expenditure Survey, 1999: Interview Survey and Detailed Expenditure Files [Computer file]. Washington, DC: U.S. Dept. of Labor, Bureau of Labor Statistics
} 
we first extract from the CEX data set only those households which contain married couples, whose tenure status is renter household or homeowner with or without mortgage, and whose age of the head is between 25 and 60. We also drop from our analysis those households whose total income was lower than $\$ 3,000$ or higher than $\$ 75,000$. (In addition, we consider households in the so-called metropolitan statistical areas because we can associate prices only to these households; see below.) Such selection, similarly used by Nicol (2001), Donald (1997), Lewbel (1991) and others, allows to have a somewhat homogeneous sample of households. With each of the selected households, we also retain the variables of interest to our study, namely, some location variables (for matching with prices) and expenditure shares $Y_{i}$ for 6 categories of goods: food, health care, transportation, household, apparel (clothing) and miscellaneous goods. The total number of households which met the above criteria was 897 (out of 7860 in the CEX data set). As in Donald (1997), Hausman et al. (1995) and others, we consider the logarithm of the income $X_{i}$, rather than the income itself.

The CEX data set contains no information on prices. We draw prices from the ACCRA data set $^{2}$ which provides a composite price index and price indices for 6 different categories of goods (grocery items, housing, utilities, transportation, health care and miscellaneous goods and services) for various cities across the U.S. We are able to associate these prices to household selected from the CEX data set by using some location variables in the CEX data set as matching variables, and also some confidential information kindly provided by the Bureau of Labor Statistics. Details on matching procedure can be obtained from the author upon request.

Though ACCRA prices are available for a few categories of goods and could be assigned for each type of expenditures considered in the demand system, we shall use only the composite price index $Z_{i}$ in our study. (For notational simplicity, we have divided the price indices $Z_{i}$ by 100 so that $z=100$ in the ACCRA data now corresponds to $z=1$.) We do so to avoid the socalled empty-space phenomenon (see, for example, pp. 59-60 in Pagan and Ullah (1999) or pp. 92-93 in Silverman (1986)): for a high-dimensional vectors $Z_{i}$, a great number of observations is needed in order to localize at a fixed value $z$. We expect that the simplest one-dimensional [producer], 2001. Ann Arbor, MI: Inter-University Consortium for Political and Social Research [distributor], 2001.

${ }^{2}$ ACCRA Cost of Living Index, Data for First Quarter 2000, ACCRA, July 2000, 33(1). For more information, see http://www.accra.org 
case becomes a guide to more general situations of multidimensional vectors $Z_{i}$ which require larger data sets. These can be constructed, for example, as in Nicol (2001), by considering the CEX and the ACCRA data for multiple quarters and using a CPI data to account for inflation in multiple quarters.

As described in Section 1, to estimate a local rank, we eliminate one share from the analysis and then add 1 to the local rank (related to $x^{1}=1$ ) of a reduced demand system estimated by the procedures of Section 4. It can be shown theoretically and is easily observed in practice that the local rank tests of Section 4 are invariant to which share is eliminated from the analysis.

Table 5 presents the $p$-values of the local rank tests for the full data set at two different values of prices $z=1$ and $z=1.2$. (We do not report the $p$-values for $L=4$ and $L=5$ because there are no results where the rank $L \leq 3$ is rejected.) These values of $z$ were motivated by the fact that prices associated with household in the constructed data set ranged from 0.911 to 1.251. The choices of the smoothing parameters $h_{x}$ and $h_{z}$ were suggested in part by the fact that $h_{x}=0.55$ and $h_{z}=0.09$ were the optimal smoothing parameters obtained by the generalized cross validation procedure for the data set consisting of all expenditure shares.

The results of Table 5 suggest that the local rank of the full demand system is 3 at $z=1$. This conclusion is reached for almost all considered values $h_{x}, h_{z}$, both tests statistics and any reasonably small significance level $\alpha$. The results also suggest that the local rank is 2 at $z=1.2$. This conclusion is more evident when the test statistic $\widehat{T}_{1}(L, z)$ is used and, in particular, it is true at the significance level $\alpha=0.05$ for both statistics when the smoothing parameter values $h_{x}=0.55$ and $h_{z}=0.09$ obtained by the generalized cross validation are used. We have also tried estimating local rank at other higher values of $z$ and for data sets of households with other characteristics. We have found in all of these experiments that estimates of local ranks tend to become smaller as z increases.

Several observations can be made from the above rank estimation results. Interestingly, different local ranks may be estimated at distinct values of prices $z$. This motivates the study of global rank tests and finding causes for the observed phenomenon which we intend to pursue in a future work. The role and significance of the rank estimation ignoring prices should also be further clarified. 


\section{Conclusions}

In the present work, we provided consistent tests to determine the local rank in nonparametric models. Two tests statistics were considered: one defined as a sum of the eigenvalues and the other defined as a sum of the squared eigenvalues of a kernel-based estimator of a matrix. The asymptotics of these statistics were based on the asymptotics of the eigenvalues which was established by using Fujikoshi expansion and $U$-statistics techniques. Simulation study showed that the local rank tests perform fairly well and that the two tests statistics have slightly different small sample properties. Our results extend those of Donald (1997) to the case where coefficient matrices vary with covariates so that distinction between local and global ranks becomes necessary. We applied our rank estimation methods to determine local ranks in a demand system constructed by combining the CEX and the ACCRA data sets. Results obtained in applications to demand systems show importance of studying global ranks.

\section{A Proofs of principal results}

Proof of Lemma 2.1: Let us show first that $\operatorname{rk}\left\{F(\cdot, z) ; x^{1}\right\} \leq L$ implies that $\Gamma_{w, z}$ has $G-L$ zero eigenvalues. By Definition 1.1, $\operatorname{rk}\left\{F(\cdot, z) ; x^{1}\right\} \leq L$ if and only if $(1.3)$ is verified. This implies that $\beta(x, z) F(x, z) x^{\prime}=$ $\beta(x, z) c(z) x^{1} x^{1^{\prime}}+A(z) \beta(x, z) H(x, z) x^{1^{\prime}}$. By substituting $X_{i}$ for $x$ and taking the expectation, we get $E\left(\beta\left(X_{i}, z\right) F\left(X_{i}, z\right) X_{i}^{1^{\prime}}\right)=$ $E\left(\beta\left(X_{i}, z\right) c(z) X_{i}^{1} X_{i}^{1^{\prime}}\right)+A(z) E\left(\beta\left(X_{i}, z\right) H\left(X_{i}, z\right) X_{i}^{1^{\prime}}\right)$. Multiplying this relation by $\left(E \beta\left(X_{i}, z\right) X_{i}^{1} X_{i}^{1^{\prime}}\right)^{-1} x^{1}$ and subtracting it from (1.3), we obtain that $\widetilde{F}(x, z)=A(z)\left(H(x, z)-E\left(\beta\left(X_{i}, z\right) H\left(X_{i}, z\right) X_{i}^{1^{\prime}}\right)\left(E \beta\left(X_{i}, z\right) X_{i}^{1} X_{i}^{1^{\prime}}\right)^{-1} x^{1}\right)$. It follows that there are $G-L$ linearly independent vectors $c_{j}(z), j=1, \ldots, G-L$, such that $c_{j}(z)^{\prime} \widetilde{F}(x, z)=0$. This is equivalent to $c_{j}(z)^{\prime} \gamma(x, z)^{1 / 2} \widetilde{F}(x, z)=0$ and $E\left(c_{j}(z)^{\prime} \gamma\left(X_{i}, z\right)^{1 / 2} \widetilde{F}\left(X_{i}, z\right)\right)^{2}=c_{j}(z)^{\prime} \Gamma_{w, z} c_{j}(z)=0$, for $j=1, \ldots, G-L$. The last relation holds if and only if the matrix $\Gamma_{w, z}$ has $G-L$ zero eigenvalues. One can, in fact, go back in the arguments above which establishes the first "if and only if" part of the lemma. The other parts of the lemma can be easily deduced.

Proof of Theorem 4.1: For shortness sake, we prove the theorem for the estimator $\widehat{\Gamma}_{w, z}$ in $(2.7)$ used for tests of local rank $\operatorname{rk}\{F(\cdot, z) ; 0\}$. (A general proof can be found in Fortuna (2004).) To simplify notation, we set $L_{z}=L(z), \widetilde{K}_{i j}=\widetilde{K}_{h}\left(X_{i}-X_{j}\right)$, $K_{z, i}=K_{h}\left(z-Z_{i}\right)$ and $\Delta F\left(x_{i}, z_{i}, z\right)=F\left(x_{i}, z_{i}\right)-F\left(x_{i}, z\right)$. By using the definition $(2.7)$ and by writing $Y_{i}=F\left(X_{i}, Z_{i}\right)+U_{i}=$ $F\left(X_{i}, z\right)+\Delta F\left(X_{i}, Z_{i}, z\right)+U_{i}$, we can express $\widehat{\Gamma}_{w, z}$ as

$$
\widehat{\Gamma}_{w, z}=\mathcal{A}_{1}+\delta \mathcal{A}_{2}+\delta^{2} \mathcal{A}_{3}=A_{1}+\delta\left(A_{2}+A_{2}^{\prime}\right)+\delta^{2}\left(A_{3}+A_{3}^{\prime}+A_{4}\right),
$$

where $\delta^{-1}=\sqrt{N h^{m+n / 2}}$, the first order term $A_{1}$ is

$$
A_{1}=\frac{1}{N(N-1)} \sum_{i \neq j}^{N} F\left(X_{i}, z\right) F\left(X_{j}, z\right)^{\prime} \widetilde{K}_{i j} K_{z, i} K_{z, j}
$$

the second order term is $A_{2}=\delta^{-1}\left(A_{2,1}+A_{2,2}\right)$ with

$$
A_{2,1}=\frac{1}{N(N-1)} \sum_{i \neq j}^{N} \Delta F\left(X_{i}, Z_{i}, z\right) F\left(X_{j}, z\right)^{\prime} \widetilde{K}_{i j} K_{z, i} K_{z, j}, A_{2,2}=\frac{1}{N(N-1)} \sum_{i \neq j}^{N} U_{i} F\left(X_{j}, z\right)^{\prime} \widetilde{K}_{i j} K_{z, i} K_{z, j},
$$

the third order term is $A_{3}=\delta^{-2}\left(A_{3,1}+A_{3,2}\right)$ with

$$
A_{3,1}=\frac{1}{2 N(N-1)} \sum_{i \neq j}^{N} \Delta F\left(X_{i}, Z_{i}, z\right) \Delta F\left(X_{j}, Z_{j}, z\right)^{\prime} \widetilde{K}_{i j} K_{z, i} K_{z, j}, A_{3,2}=\frac{1}{N(N-1)} \sum_{i \neq j}^{N} \Delta F\left(X_{i}, Z_{i}, z\right) U_{j}^{\prime} \widetilde{K}_{i j} K_{z, i} K_{z, j}
$$


and

$$
A_{4}=\frac{\delta^{-2}}{N(N-1)} \sum_{i \neq j}^{N} U_{i} U_{j}^{\prime} \widetilde{K}_{i j} K_{z, i} K_{z, j}
$$

The eigenvalues of $\widehat{\Gamma}_{w, z} \widehat{\Sigma}^{-1}$ are also the eigenvalues of $J^{\prime} \widehat{\Gamma}_{w, z} J\left(J^{\prime} \widehat{\Sigma} J\right)^{-1}$, where $J$ is any orthogonal matrix. The idea is to take a special $J$ which would allow for easier manipulations later. In order to choose such $J$, observe by Lemma B.1 below that $A_{1} \Sigma^{-1}$ has $G-L_{z}$ zero eigenvalues and the remaining ones are strictly positive with probability approaching 1 . Since we need to show convergence in distribution, we may suppose without loss of generality that all the eigenvalues of $A_{1} \Sigma^{-1}$ are positive. Then, there is an orthogonal matrix $J=J(N, z)$ such that the matrix $J^{\prime} A_{1} \Sigma^{-1} J=J^{\prime} A_{1} J\left(J^{\prime} \Sigma J\right)^{-1}$ is diagonal with the eigenvalues of $A_{1} \Sigma^{-1}$ on the diagonal. Since $\Sigma$ is positive definite, there is an orthogonal matrix $J_{0}$ such that $J_{0}^{\prime} \Sigma J_{0}=C$, where $C$ is a diagonal matrix. We will suppose without loss of generality that $C=I$ and hence that $J_{0}^{\prime} \Sigma J_{0}=I$. Since there is an orthogonal matrix $J_{1}$ such that $J_{0} J_{1}=J$, we have $J^{\prime} \Sigma J=J_{1}^{\prime} J_{0}^{\prime} \Sigma J_{0} J_{1}=J_{1}^{\prime} J_{1}=I$. The discussion above implies that $J^{\prime} A_{1} J$ is diagonal with $G-L_{z}$ zeros on the diagonal and the remaining elements on the diagonal strictly positive (with probability approaching 1 ). We can then arrange $J$ as $J=\left(\begin{array}{lll}J_{1} & J_{2}\end{array}\right)$, where $J_{1}$ is $G \times L_{z}$ and $J_{2}$ is $G \times\left(G-L_{z}\right)$, in such a way that $J_{2}^{\prime} A_{1} J_{2}=0$. Since $J_{2}$ consists of eigenvectors corresponding to zero eigenvalues of $A_{1}$, it follows from Lemma B.1 below that $A_{2} J_{2}=0$ and hence that $J^{\prime} A_{2} J$ has its last $G-L_{z}$ columns identically zero. Similarly, the last $G-L_{z}$ rows of $J^{\prime} A_{2}^{\prime} J$ are identically zero as well. Finally, observe that the effect of $J$ 's on the term $A_{4}$ is now such that $E\left(J^{\prime} U_{i} U_{i}^{\prime} J\right)=I$.

By using $\widehat{\Sigma}=\Sigma+\delta B$ with $B=o_{p}(1)$ in Lemma B.7 below, $A_{i}=O_{p}(1), i=1, \ldots, 4$, in Lemmas B.2-B.5 below and the discussion above, $\delta^{-2} \widehat{\lambda}_{j}(z)$ is equal to $\delta^{-2}$ times the $j$ th smallest eigenvalue of the matrix

$$
\begin{aligned}
J^{\prime} \widehat{\Gamma}_{w, z} J\left(J^{\prime} \widehat{\Sigma} J\right)^{-1} & =J^{\prime} \widehat{\Gamma}_{w, z} J\left(J^{\prime} \Sigma J+\delta J^{\prime} B J\right)^{-1}=J^{\prime} \widehat{\Gamma}_{w, z} J\left(I+\delta J^{\prime} B J\right)^{-1} \\
& =J^{\prime}\left(\mathcal{A}_{1}+\delta \mathcal{A}_{2}+\delta^{2} \mathcal{A}_{3}\right) J\left(I-\delta J^{\prime} B J+\delta^{2} J^{\prime} B^{2} J-\ldots\right)=D_{1}+\delta D_{2}+\delta^{2} D_{3}+O_{p}\left(\delta^{3}\right),
\end{aligned}
$$

where $D_{1}=J^{\prime} \mathcal{A}_{1} J=J^{\prime} A_{1} J$ is diagonal, $D_{2}=J^{\prime}\left(\mathcal{A}_{2}-\mathcal{A}_{1} B\right) J=O_{p}(1)$ and $D_{3}=J^{\prime}\left(\mathcal{A}_{3}-\mathcal{A}_{2} B+\mathcal{A}_{1} B^{2}\right) J=O_{p}(1)$. By applying Lemma 1 in Fujikoshi (1977), we can conclude that $\widehat{\lambda}_{j}(z), j=1, \ldots, G-L_{z}$, are also the eigenvalues of the matrix

$$
0 I+\delta \widetilde{D}_{2}+\delta^{2} \widetilde{D}_{3}+O_{p}\left(\delta^{3}\right)
$$

where the matrices $\widetilde{D}_{2}$ and $\widetilde{D}_{3}$ are described in greater detail below.

The matrix $\widetilde{D}_{2}$ in (A.2) is a $\left(G-L_{z}\right) \times\left(G-L_{z}\right)$ matrix made of the last $G-L_{z}$ rows and the last $G-L_{z}$ columns of the matrix $D_{2}=J^{\prime} \mathcal{A}_{2} J-J^{\prime} \mathcal{A}_{1} B J$. Recall from (A.1) and the discussion above that $J^{\prime} \mathcal{A}_{2} J$ is a sum of two matrices $J^{\prime} A_{2} J$ and $J^{\prime} A_{2}^{\prime} J$, the matrix $J^{\prime} A_{2} J$ with its last $G-L_{z}$ columns zero and the matrix $J^{\prime} A_{2}^{\prime} J$ with its last $G-L_{z}$ rows zero. Hence, the $\left(G-L_{z}\right) \times\left(G-L_{z}\right)$ matrix corresponding to $J^{\prime} \mathcal{A}_{2} J$ is identically zero. Turning to the second term $J^{\prime} \mathcal{A}_{1} B J=J^{\prime} \mathcal{A}_{1} J\left(J^{\prime} B J\right)$ in the matrix $D_{2}$, since $J^{\prime} \mathcal{A}_{1} J$ is diagonal with its last $G-L_{z}$ rows zero, we obtain that the $\left(G-L_{z}\right) \times\left(G-L_{z}\right)$ matrix corresponding to $J^{\prime} \mathcal{A}_{1} B J$ is identically zero as well. Then, $\widetilde{D}_{2}=0$ and hence $\widehat{\lambda}_{j}(z), j=1, \ldots, G-L_{z}$, are also the eigenvalues of the matrix $\delta^{2} \widetilde{D}_{3}+O_{p}\left(\delta^{3}\right)$ or $\delta^{-2} \widehat{\lambda}_{j}(z)=N h^{m+n / 2} \widehat{\lambda}_{j}(z), j=1, \ldots, G-L_{z}$, are the eigenvalues of the matrix

$$
\widetilde{D}_{3}+o_{p}(1) \text {. }
$$

According to Lemma 1 in Fujikoshi (1977), the matrix $\widetilde{D}_{3}$ in (A.3) (or (A.2)) is a sum of two matrices $\widetilde{D}_{3,1}$ and $\widetilde{D}_{3,2}$. The first term $\widetilde{D}_{3,1}$ is made of the last $G-L_{z}$ rows and the last $G-L_{z}$ columns of the matrix $D_{3}$. The second term $\widetilde{D}_{3,2}$ involves the sum of some submatrices of the last $G-L_{z}$ rows and the last $G-L_{z}$ columns of the matrix $D_{2}$. By using the facts that $A_{2}=o_{p}(1), B=o_{p}(1)$ and a special structure of the matrix $J^{\prime} A_{1} J$, we can conclude that $\widetilde{D}_{3,2}=o_{p}(1)$. As for the matrix $\widetilde{D}_{3,1}$, by using $A_{3}=o_{p}(1)$, we obtain that it consists of the last $G-L_{z}$ rows and the last $G-L_{z}$ columns of the matrix

$$
J^{\prime} A_{4} J+o_{p}(1)=\frac{\delta^{-2}}{N(N-1)} \sum_{i \neq j}^{N}\left(J^{\prime} U_{i}\right)\left(J^{\prime} U_{j}\right)^{\prime} \widetilde{K}_{i j} K_{z, i} K_{z, j}+o_{p}(1) .
$$

Hence, it follows that

$$
\widetilde{D}_{3}=\frac{\delta^{-2}}{N(N-1)} \sum_{i \neq j}^{N} \widetilde{U}_{i} \widetilde{U}_{j}^{\prime} \widetilde{K}_{i j} K_{z, i} K_{z, j}+o_{p}(1),
$$

where a $\left(G-L_{z}\right) \times 1$ vector $\widetilde{U}_{j}$ satisfies $E \widetilde{U}_{j} \widetilde{U}_{j}^{\prime}=I$. By Lemma B.6 below, we have $\widehat{V}(z) \widetilde{D}_{3} \stackrel{d}{\rightarrow} \mathcal{Z}_{G-L_{0}(z)}$. The convergence $(4.4)$ then follows from this and (??) by the continuous mapping theorem.

The convergence (4.5) holds, since by the continuous mapping theorem, $\widehat{\lambda}_{j}(z) \rightarrow \lambda_{j}(z)$ in probability, where $0 \leq \lambda_{1}(z) \leq \ldots \leq$ $\lambda_{G}(z)$ are the eigenvalues of the matrix $\Gamma_{w, z} \Sigma^{-1}$ and, by Lemma $2.1, \lambda_{j}(z)>0$ for $j=G-L_{z}+1, \ldots, G$.

Proof of Theorem 4.2: The convergence in (4.8) and (4.9) follows from (4.4) in Theorem 4.1. The stochastic dominance (4.8) can be proved as in Theorem 2 of Donald (1997). To prove the stochastic dominance in (4.9), observe first that 
$\sum_{j=1}^{G-L}\left(\lambda_{j}\left(\mathcal{Z}_{G-L_{z}}\right)\right)^{2}=\sum_{j=1}^{G-L} \lambda_{j}\left(\mathcal{Z}_{G-L_{z}}^{2}\right)$, where $\lambda_{j}\left(\mathcal{Z}_{G-L_{z}}^{2}\right), j=1, \ldots, G-L_{z}$, denote the eigenvalues of $\mathcal{Z}_{G-L_{z}}^{2}$ in the increasing order, and $L_{z}=\operatorname{rk}\left\{F(\cdot, z) ; x^{1}\right\}$. Take $B=\left(0_{(G-L) \times(L-L z)} I_{G-L}\right)^{\prime}$ so that $B^{\prime} B=I_{G-L}$. By the Poincaré separation theorem (see Magnus and Neudecker (1999), p. 209, or Rao (1973), p. 65), we have $\lambda_{i}\left(\mathcal{Z}_{G-L_{z}}\right) \leq \lambda_{i}\left(B^{\prime} \mathcal{Z}_{G-L_{z}} B\right)$ for $i=1, \ldots, G-L$. Then $\sum_{j=1}^{G-L} \lambda_{j}\left(\mathcal{Z}_{G-L_{z}}^{2}\right) \leq_{d} \sum_{j=1}^{G-L} \lambda_{j}\left(\left(B^{\prime} \mathcal{Z}_{G-L_{z}} B\right)\left(B^{\prime} \mathcal{Z}_{G-L_{z}} B\right)\right)$. Since $B^{\prime} \mathcal{Z}_{G-L_{z}} B={ }_{d} \mathcal{Z}_{G-L}$, it follows from above that $\sum_{j=1}^{G-L}\left(\lambda_{j}\left(\mathcal{Z}_{G-L_{z}}\right)\right)^{2} \leq_{d} \sum_{j=1}^{G-L} \lambda_{j}\left(\mathcal{Z}_{G-L}^{2}\right)=\operatorname{tr}\left\{\mathcal{Z}_{G-L}^{2}\right\}=\operatorname{vec}\left(\mathcal{Z}_{G-L}\right)^{\prime} \operatorname{vec}\left(\mathcal{Z}_{G-L}\right)=_{d} \chi^{2}((G-L)(G-L+1) / 2)$, since $\mathcal{Z}_{G-L}$ is a symmetric matrix consisting of independent (below the diagonal) zero mean normal random variables with variance 1 on the diagonal and variance $1 / 2$ off the diagonal (use the fact $\left.2(\mathcal{N}(0,1 / 2))^{2}={ }_{d} \mathcal{N}(0,1)^{2}\right)$.

Proof of Theorem 4.3: Consider first the case $i=1$. The proof is similar to that of Theorem 3 in Donald (1997) or Theorem 5.2 in Robin and Smith (2000). Let $\mathcal{A}_{L}$ denote the event that the null hypothesis $H_{0}: \operatorname{rk}\left\{F(\cdot, z) ; x^{1}\right\} \leq L$ is rejected by using the statistic $\widehat{T}_{1}(L, z)$ at the significance level $\alpha=\alpha(N, h)$. Then, we have

$$
P\left(\widehat{L}_{1}(z)=L\right)=P\left(\mathcal{A}_{1} \cap \ldots \cap \mathcal{A}_{L-1} \cap \mathcal{A}_{L}^{c}\right),
$$

where $\mathcal{A}_{L}^{c}$ denotes the complement of $\mathcal{A}_{L}$. Let $\mathcal{N}_{\alpha(N, h)}(0,1)$ be the minimum $\xi$ such that $P(\mathcal{N}(0,1)>\xi)=\alpha(N, h)$. Obviously, $\mathcal{N}_{\alpha(N, h)}(0,1) \rightarrow \infty$ if $\alpha(N, h) \rightarrow 0$. It is also an easy exercise to see that $\mathcal{N}_{\alpha(N, h)}(0,1) / N h^{m+n / 2} \rightarrow 0$ if $(-\ln \alpha(N, h))^{1 / 2} / N h^{m+n / 2} \rightarrow 0$. Then, for any $L<\operatorname{rk}\left\{F(\cdot, z) ; x^{1}\right\}$, we obtain from (A.4) that

$$
P\left(\widehat{L}_{1}(z)=L\right) \leq P\left(\mathcal{A}_{L}^{c}\right)=1-P\left(\widehat{T}_{1}(L, z)>\mathcal{N}_{\alpha(N, h)}(0,1)\right)=1-P\left(\widehat{T}_{1}(L, z) / N h^{m+n / 2}>\mathcal{N}_{\alpha(N, h)}(0,1) / N h^{m+n / 2}\right) \rightarrow 0,
$$

by using $\widehat{T}_{1}(L, z) / N h^{m+n / 2} \rightarrow$ Const $>0$ and $\mathcal{N}_{\alpha(N, h)}(0,1) / N h^{m+n / 2} \rightarrow \infty$. Observe also that, by setting $L_{z}=\operatorname{rk}\left\{F(\cdot, z) ; x^{1}\right\}$, we have

$$
P\left(\widehat{L}_{1}(z)>L_{z}\right) \leq P\left(\mathcal{A}_{L_{z}}\right)=P\left(\widehat{T}_{1}\left(L_{z}, z\right)>\mathcal{N}_{\alpha(N, h)}(0,1)\right) \rightarrow 0
$$

by using Theorem 4.2 and since $\mathcal{N}_{\alpha(N, h)}(0,1) \rightarrow 0$. The convergence in (A.5) and (A.6) show that $P\left(\widehat{L}_{1}(z)=L_{z}\right) \rightarrow 1$. When $i=2$, introduce $\chi_{\alpha(N, h)}^{2}((G-L)(G-L+1) / 2)$ as the minimum $\xi$ such that $P\left(\chi^{2}((G-L)(G-L+1) / 2)>\xi\right)=\alpha(N, h)$. Observe that, by Theorem 5.8 in Pötscher (1983), we have $\chi_{\alpha(N, h)}^{2}((G-L)(G-L+1) / 2) \rightarrow \infty$ if $\alpha(N, h) \rightarrow 0$ and $\chi_{\alpha(N, h)}^{2}((G-L)(G-L+1) / 2) \rightarrow 0$ if $-\ln \alpha(N, h) / N^{2} h^{2 m+n} \rightarrow 0$. The rest of the argument is the same as in the case $i=1$.

\section{B Intermediate results}

We first prove two elementary results used in Theorem 4.1 in Appendix A.

Lemma B.1 The matrix $A_{1}$ (or the matrix $A_{1} \Sigma^{-1}$ ) in $(A .1)$ has $G-L_{z}$ zero eigenvalues and the remaining ones are positive with probability approaching 1 . The eigenvectors corresponding to $G-L_{z}$ zero eigenvalues of the matrix $A_{1}$ are also eigenvectors for the matrix $A_{2}$ in (A.1) corresponding to a zero eigenvalue.

Proof: By using (1.3), $F\left(X_{i}, z\right)=A(z) H\left(X_{i}, z\right)$. Substituting this into (A.1), we obtain $A_{1}=A(z) H_{1} A(z)^{\prime}$, where $H_{1}=$ $(N(N-1))^{-1} \sum_{i \neq j}^{N} H\left(X_{i}, z\right) H\left(X_{j}, z\right)^{\prime} \widetilde{K}_{i j} K_{z, i} K_{z, j}$. Since $A(z)$ is a $G \times L_{z}$ matrix, there are $G-L_{z}$ linearly independent vectors $c_{j}(z)$ such that $c_{j}(z) A(z)=0$. Then, $A_{1} c_{j}(z)^{\prime}=A(z) H_{1} A(z)^{\prime} c_{j}(z)^{\prime}=0$ for $j=1, \ldots, G-L_{z}$, which shows that $A_{1}$ has $G-L_{z}$ zero eigenvalues. The remaining eigenvalues are positive with probability approaching 1 because $A_{1} \rightarrow_{p} \Gamma_{w, z}$ (Lemma B.2 below) and the matrix $\Gamma_{w, z}$ has $G-L_{z}$ zero eigenvalues with the remaining ones strictly positive (Lemma 2.1 above). The second statement of the lemma can be proved by using similar arguments. $\square$

The next four lemmas concern the orders of the terms $A_{1}, A_{2}, A_{3}$ and $A_{4}$ in the decomposition (A.1). Their proofs often use the notion of a second order $U$-statistic whose definition we recall in Appendix C, together with a useful result on their asymptotic behavior.

Lemma B.2 Under the assumptions of Theorem 4.1, we have $A_{1}=\Gamma_{w, z}+o_{p}(1)$.

ProOF: We need to show that

$$
A_{1}=E\left(\frac{p\left(X_{i}, z\right)^{2}}{\widetilde{p}\left(X_{i}\right)} F\left(X_{i}, z\right) F\left(X_{i}, z\right)^{\prime}\right)+o_{p}(1)
$$

We will prove (B.1) in the case $G=1$. The case $G \geq 2$ follows by considering matrices component-wise. Observe that $A_{1}$ can be expressed as a second order $U$-statistic (C.1) with $W_{i}=\left(X_{i}, Z_{i}\right)$ and $a_{N}\left(W_{i}, W_{j}\right)=F\left(X_{i}, z\right) F\left(X_{j}, z\right) \widetilde{K}_{i j} K_{z, i} K_{z, j}$. By using the assumptions of Theorem 4.1 and applying Lemma B.9, $(a)$, below, we have

$$
\begin{gathered}
E a_{N}\left(W_{i}, W_{j}\right)=E\left(\frac{p\left(X_{i}, z\right)^{2}}{\widetilde{p}\left(X_{i}\right)} F\left(X_{i}, z\right) F\left(X_{i}, z\right)\right)+o(1), \\
E\left(E\left(a_{N}\left(W_{i}, W_{j}\right) \mid W_{i}\right)^{2}\right)=O\left(h^{-m}\right)=o(N), \quad E a_{N}\left(W_{i}, W_{j}\right)^{2}=O\left(h^{-2 m-n}\right)=o\left(N^{2}\right),
\end{gathered}
$$

since $N h^{m} \rightarrow \infty$ and $N h^{m+n / 2} \rightarrow \infty$. The relation (B.1) then follows from Lemma C.1 below. 
Lemma B.3 Under the assumptions of Theorem 4.1, we have $A_{2}=o_{p}(1)$.

Proof: We will argue that

$$
A_{2,1}=O_{p}\left(h^{r}+\sqrt{\frac{h^{2}}{N h^{m}}}+\sqrt{\frac{h^{2}}{N^{2} h^{2 m+n}}}\right), \quad A_{2,2}=O_{p}\left(\frac{1}{\sqrt{N h^{m}}}+\frac{1}{\sqrt{N^{2} h^{2 m+n}}}\right)
$$

where $A_{2, i}, i=1,2$, are defined after (A.1). Then, the order of $A_{2}$ can be seen to be $O_{p}\left(\sqrt{N h^{m+n / 2+2 r}}+\sqrt{h^{n / 2}}+1 / \sqrt{N h^{m+n / 2}}\right)=$ $o_{p}(1)$ since $N h^{m+n / 2+2 r} \rightarrow 0$ and $N h^{m+n / 2} \rightarrow \infty$. Consider the first relation in (B.2) and suppose for simplicity that $G=1$. Observe that $A_{2,1}$ is a second order $U$-statistic (C.1) with $W_{i}=\left(X_{i}, Z_{i}\right)$ and $a_{N}\left(W_{i}, W_{j}\right)=2^{-1}\left(\Delta F\left(X_{i}, Z_{i}, z\right) F\left(X_{j}, z\right)+\right.$ $\left.\Delta F\left(X_{j}, Z_{j}, z\right) F\left(X_{i}, z\right)\right) \widetilde{K}_{i j} K_{z, i} K_{z, j}=: a_{N, 1}\left(W_{i}, W_{j}\right)+a_{N, 2}\left(W_{i}, W_{j}\right)$. By using the assumptions of Theorem 4.1 and applying Lemma B.9, $(a)$, below, we get $E a_{N}\left(W_{i}, W_{j}\right)=O\left(h^{r}\right)$ and

$$
\begin{gathered}
E\left(E\left(a_{N}\left(W_{i}, W_{j}\right) \mid W_{i}\right)^{2}\right) \leq 2 E\left(E\left(a_{N, 1}\left(W_{i}, W_{j}\right) \mid W_{i}\right)^{2}\right)+2 E\left(E\left(a_{N, 2}\left(W_{i}, W_{j}\right) \mid W_{i}\right)^{2}\right)=O\left(h^{2 r-m}+h^{2-m}\right)=O\left(h^{2-m}\right) \\
E a_{N}\left(W_{i}, W_{j}\right)^{2} \leq 2 E a_{N, 1}\left(W_{i}, W_{j}\right)^{2}+2 E a_{N, 2}\left(W_{i}, W_{j}\right)^{2}=O\left(\frac{h^{2}}{h^{2 m+n}}\right)
\end{gathered}
$$

The first relation of (B.2) then follows from Lemma C.1 below. In the case of the second relation of (B.2), supposing for simplicity that $G=1, A_{2,2}$ is a second order $U$-statistic with $W_{i}=\left(Y_{i}, X_{i}, Z_{i}\right)$ and $a_{N}\left(W_{i}, W_{j}\right)=2^{-1}\left(U_{i} F\left(X_{j}, z\right)+\right.$ $\left.U_{j} F\left(X_{i}, z\right)\right) \widetilde{K}_{i j} K_{z, i} K_{z, j}=: a_{N, 1}\left(W_{i}, W_{j}\right)+a_{N, 2}\left(W_{i}, W_{j}\right)$. By using the assumptions of Theorem 4.1 and applying Lemma B.9, $(a)$, below again, we have $E a_{N}\left(W_{i}, W_{j}\right)=0, E\left(E\left(a_{N}\left(W_{i}, W_{j}\right) \mid W_{i}\right)^{2}\right)=E U_{i}^{2}\left(E\left(a_{N, 1}\left(W_{i}, W_{j}\right) \mid W_{i}\right)^{2}\right)=O\left(h^{-m}\right)$ and $E a_{N}\left(W_{i}, W_{j}\right)^{2}=E a_{N, 1}\left(W_{i}, W_{j}\right)^{2}+E a_{N, 2}\left(W_{i}, W_{j}\right)^{2}=O\left(h^{-2 m-n}\right)$. The conclusion follows from Lemma C.1 below.

Lemma B.4 Under the assumptions of Theorem 4.1, we have $A_{3}=o_{p}(1)$.

ProOF: We will argue that

$$
A_{3,1}=O_{p}\left(h^{2 r}+\sqrt{\frac{h^{2 r+2}}{N h^{m}}}+\sqrt{\frac{h^{4}}{N^{2} h^{2 m+n}}}\right), \quad A_{3,2}=O_{p}\left(\sqrt{\frac{h^{2 r}}{N h^{m}}}+\sqrt{\frac{h^{2}}{N^{2} h^{2 m+n}}}\right)
$$

where $A_{3,1}$ and $A_{3,2}$ are defined after (A.1). Then, we can deduce that $A_{3}=O_{p}\left(\sqrt{N h^{m+n / 2+2 r}}+h\right)=o_{p}(1)$ since $N h^{m+n / 2+2 r} \rightarrow 0$. Supposing for simplicity that $G=1, A_{3,1}$ is a second order $U$-statistic $\left(\right.$ C.1) with $W_{i}=\left(X_{i}, Z_{i}\right)$ and $a_{N}\left(W_{i}, W_{j}\right)=\Delta F\left(X_{i}, Z_{i}, z\right) \Delta F\left(X_{j}, Z_{j}, z\right) \widetilde{K}_{i j} K_{z, i} K_{z, j}$. Then, by using the assumptions of Theorem 4.1 and arguing as in the proof of Lemma B.9, $(a)$, below, we have $E a_{N}\left(W_{i}, W_{j}\right)=O\left(h^{2 r}\right), E\left(E\left(a_{N}\left(W_{i}, W_{j}\right) \mid W_{i}\right)^{2}\right)=O\left(h^{2 r+2-m}\right)$ and $E a_{N}\left(W_{i}, W_{j}\right)^{2}=O\left(h^{4-2 m-n}\right)$. The first relation in (B.3) then follows by using Lemma C.1. As for $A_{3,2}$, it is a second order $U$ statistic (C.1) with $W_{i}=\left(Y_{i}, X_{i}, Z_{i}\right)$ and $a_{N}\left(W_{i}, W_{j}\right)=2^{-1}\left(\Delta F\left(X_{i}, Z_{i}, z\right) U_{j}+\Delta F\left(X_{j}, Z_{j}, z\right) U_{i}\right) \widetilde{K}_{i j} K_{z, i} K_{z, j}$. By the assumptions of Theorem 4.1, $E a_{N}\left(W_{i}, W_{j}\right)=0$ and, by using Lemma B.9, $(a)$, below, we can show that $E\left(E\left(a_{N}\left(W_{i}, W_{j}\right) \mid W_{i}\right)^{2}\right)=O\left(h^{2 r-m}\right)$ and $E a_{N}\left(W_{i}, W_{j}\right)^{2}=O\left(h^{2-2 m-n}\right)$. The second relation in (B.3) follows from Lemma C.1 below.

Lemma B.5 Under the assumptions of Theorem 4.1, we have $A_{4}=O_{p}(1)$.

ProOF: Arguing as in the proof of Lemma B.6 below, we may show that $A_{4}$ is asymptotically normal and hence, $A_{4}=O_{p}(1)$.

We now prove an asymptotic normality result used in the proof of Theorem 4.1.

Lemma B.6 Under the assumptions and with the notation of Theorem 4.1 and its proof, we have

$$
\widehat{V}(z) \frac{h^{m+n / 2}}{N} \sum_{i \neq j}^{N} \widetilde{U}_{i} \widetilde{U}_{j}^{\prime} \widetilde{K}_{i j} K_{z, i} K_{z, j} \stackrel{d}{\rightarrow} \mathcal{Z}_{G-L_{z}} .
$$


Proof: Set $t=G-L_{z}, \widetilde{U}_{i}=\left(\widetilde{U}_{i 1}, \ldots, \widetilde{U}_{i t}\right)^{\prime}$ and $A_{p, q}(N)=h^{m+n / 2} N^{-1} \sum_{i \neq j}^{N} \widetilde{U}_{i p} \widetilde{U}_{j q} \widetilde{K}_{i j} K_{z, i} K_{z, j}, p, q=1, \ldots, t$, so that the left-hand side of (B.4) can be expressed as $\widehat{V}(z)\left(A_{p, q}(N)\right)_{p, q=1, \ldots, t}$. We will show first that, for fixed $p$ and $q$,

$$
A_{p, q}(N) \stackrel{d}{\rightarrow} \mathcal{N}\left(0, \sigma_{p, q}^{2} V(z)^{-2}\right)
$$

where $V(z)$ is defined in the beginning of Section 4 and $\sigma_{p, q}^{2}=1$, if $p=q$, and $1 / 2$, if $p \neq q$. By using Lemma B.8 below, the convergence (B.4) then holds component-wise.

To show (B.5), we follow the proof of Theorem 4.5 in White and Hong (1999) (see also Lemma B.2 in Donald (1997)) Since $\widetilde{U}_{i}$ can be expressed in terms of $W_{i}=\left(Y_{i}, X_{i}, Z_{i}\right)$, we can write $A_{p, q}(N)=\sum_{i \neq j}^{N} \widetilde{a}_{N}\left(W_{i}, W_{j}\right)=\sum_{i<j} a_{N}\left(W_{i}, W_{j}\right)$, where $\widetilde{a}_{N}\left(W_{i}, W_{j}\right)=h^{m+n / 2} N^{-1} \widetilde{U}_{i p} \widetilde{U}_{j q} \widetilde{K}_{i j} K_{z, i} K_{z, j}$ and $a_{N}\left(W_{i}, W_{j}\right)=\widetilde{a}_{N}\left(W_{i}, W_{j}\right)+\widetilde{a}_{N}\left(W_{j}, W_{i}\right)$. Observe that, for $i<j$, $E\left(a_{N}\left(W_{i}, W_{j}\right) \mid W_{i}\right)=0$. Hence, by Proposition 3.2 in de Jong (1987), convergence (B.5) holds if $(1) \operatorname{Var}\left(A_{p, q}(N)\right) \rightarrow \sigma_{p, q}^{2}$, and $(2)$ $G_{N, i}=o\left(\operatorname{Var}\left(A_{p, q}(N)\right)^{2}\right)=o(1)$ for $i=1,2$ and 4, where

$$
\begin{gathered}
G_{N, 1}=\sum_{1 \leq i<j \leq N} E a_{i j}^{4}, \quad G_{N, 2}=\sum_{1 \leq i<j<k \leq N}\left(E a_{i j}^{2} a_{i k}^{2}+E a_{j i}^{2} a_{j k}^{2}+E a_{k i}^{2} a_{k j}^{2}\right), \\
G_{N, 4}=\sum_{1 \leq i<j<k<l \leq N}\left(E a_{i j} a_{i k} a_{l j} a_{l k}+E a_{i j} a_{i l} a_{k j} a_{k l}+E a_{i k} a_{i l} a_{j k} a_{j l}\right) \quad \text { with } a_{i j}=a_{N}\left(W_{i}, W_{j}\right) .
\end{gathered}
$$

To show part (1), observe that, by using Lemma B.9,(a), below,

$$
\operatorname{Var}\left(A_{p, q}(N)\right)=2 \sigma_{p, q}^{2} \frac{h^{2 m+n}}{N^{2}}(N-1) N E\left(\widetilde{K}_{i j} K_{z, i} K_{z, j}\right)^{2}=\sigma_{p, q}^{2} 2\|\widetilde{K}\|_{2}^{2}\|K\|_{2}^{4} E \frac{p\left(X_{i}, z\right)^{2}}{\widetilde{p}\left(X_{i}\right)}+o(1)=\sigma_{p, q}^{2} V(z)^{-2}+o(1) .
$$

As for part (2), by using the Lemma B.9,(b), below,

$$
\begin{gathered}
G_{N, 1} \leq \mathrm{Const} \frac{h^{4 m+2 n}}{N^{4}} \sum_{i \neq j} E\left(\widetilde{K}_{i j}^{4} K_{z, i}^{4} K_{z, j}^{4}\right) \leq \operatorname{Const} \frac{h^{4 m+2 n}}{N^{2}} E\left(\widetilde{K}_{i j}^{4} K_{z, i}^{4} K_{z, j}^{4}\right)=O\left(\frac{1}{N^{2} h^{2 m+n}}\right)=o(1), \\
G_{N, 2} \leq \operatorname{Const} \frac{h^{4 m+2 n}}{N} E\left(\widetilde{K}_{i j}^{2} \widetilde{K}_{i l}^{2} K_{z, i}^{4} K_{z, j}^{2} K_{z, l}^{2}\right)=O\left(\frac{1}{N h^{m}}\right)=o(1), \\
G_{N, 4} \leq \operatorname{Const} h^{4 m+2 n} E\left(\widetilde{K}_{i j} \widetilde{K}_{i k} \widetilde{K}_{l j} \widetilde{K}_{l k} K_{z, i}^{2} K_{z, j}^{2} K_{z, k}^{2} K_{z, l}^{2}\right)=O\left(h^{2 n}\right)=o(1) .
\end{gathered}
$$

Arguing similarly as above, we may show that, for any $c_{j} \in \mathbb{R}, p_{j}, q_{j} \in\{1, \ldots, t\}$, a linear combination $\sum_{j=1}^{d} c_{j} A_{p_{j}, q_{j}}(N)$ is asymptotically normal with the limiting variance $\sigma(\boldsymbol{p}, \boldsymbol{q})^{2}$ characterized by $\operatorname{Var}\left(\sum_{j=1}^{d} c_{j} A_{p_{j}, q_{j}}(N)\right) \rightarrow \sigma(\boldsymbol{p}, \boldsymbol{q})^{2}$. Since $E A_{p, q}(N) A_{p^{\prime}, q^{\prime}}(N)=0$ for different pairs $(p, q)$ and $\left(p^{\prime}, q^{\prime}\right)$, we conclude that $\sigma(\boldsymbol{p}, \boldsymbol{q})^{2}=\sigma_{p_{1}, q_{1}}^{2}+\ldots+\sigma_{p_{d}, q_{d}}^{2}$. Together with the convergence (B.5), this shows that (B.4) holds.

The next two results were used in the proof of Theorem 4.1 to replace the covariance matrix $\Sigma$ and a normalizing constant $V(z)$ by their estimators $\widehat{\Sigma}$ and $\widehat{V}(z)$, respectively. (See (4.1) and (4.2) for definitions of $V(z)$ and $\widehat{V}(z)$, respectively.)

Lemma B.7 Under the assumptions of Theorem 4.1, we have $\widehat{\Sigma}=\Sigma+\delta B$ with $B=o_{p}(1)$.

Proof: As shown in the proof of Lemma 2 in Donald (1997), pp. 126-127, $\widehat{\Sigma}=\Sigma+O_{p}\left(1 / \sqrt{N}+1 / N h^{m+n}+h^{2 r}\right)$. By using the assumptions of Theorem 4.1 and since $\delta^{-1}=\sqrt{N h^{m+n / 2}}$, we obtain that $\widehat{\Sigma}=\Sigma+\delta B$ with $B=o_{p}(1)$.

Lemma B.8 Under the assumptions of Theorem 4.1, we have $\widehat{V}(z)=V(z)+o_{p}(1)$.

Proof: In view of (4.1) and (4.2), it is enough to show that $N^{-1} \sum_{i=1}^{N} \widehat{p}\left(X_{i}, Z_{i}\right) K_{h}\left(z-Z_{i}\right)$ converges in probability to $E p\left(X_{i}, z\right)^{2} / \widetilde{p}\left(X_{i}\right)$. Setting $K_{i j}=K_{h}\left(Z_{i}-Z_{j}\right)$ and using $(2.10)$, we can write the sum above as

$$
\frac{1}{N^{2}} \sum_{i, j} \widetilde{K}_{i j} K_{z, i} K_{i j}=\frac{\widetilde{K}(0) K(0)}{N h^{m+n}} \frac{1}{N} \sum_{i} K_{z, i}+\frac{1}{N^{2}} \sum_{i \neq j}^{N} \widetilde{K}_{i j} K_{z, i} K_{i j}=: I_{1}+I_{2} .
$$

It is easy to show that $N^{-1} \sum_{i} K_{z, i}=O_{p}(1)$. Since $N h^{m+n} \rightarrow \infty$, it follows that $I_{1}=o_{p}(1)$. Arguing as in the proof of Lemma B.2, we obtain that $I_{2}=E\left(\widetilde{K}_{i j} K_{z, i} K_{i j}\right)+o_{p}(1)$. By using Lemma B.9, $(a)$, below we have $E\left(\widetilde{K}_{i j} K_{z, i} K_{i j}\right)=E\left(p\left(X_{i}, z\right)^{2} / \widetilde{p}\left(X_{i}\right)\right)+o(1)$ which concludes the proof.

The next result, used a number of times earlier, is a direct consequence of a localization property of kernel functions stated in Proposition B.1 below. We use our earlier notation $\widetilde{K}_{i j}=\widetilde{K}_{h}\left(X_{i}-X_{j}\right), K_{z, i}=\widetilde{K}_{h}\left(z-Z_{i}\right)$ and $K_{i j}=K_{h}\left(Z_{i}-Z_{j}\right)$. 
Lemma B.9 Suppose that $G, H: \mathbb{R}^{n} \times \mathbb{R}^{m} \times \mathbb{R}^{m} \rightarrow \mathbb{R}$ are two deterministic functions with continuous bounded derivatives up to order $r, K$ and $\widetilde{K}$ are kernels functions of order $r$ and $\left(X_{i}, Z_{i}\right)$ are i.i.d. random vectors satisfying, Assumption 1 of Section 3. Then, (a) for $i \neq j$,

$$
\begin{gathered}
E\left(G\left(X_{i}, Z_{i}, z\right) H\left(X_{j}, Z_{j}, z\right) \widetilde{K}_{i j} K_{z, i} K_{z, j}\right)=E\left(G\left(X_{i}, z, z\right) H\left(X_{i}, z, z\right) \frac{p\left(X_{i}, z\right)^{2}}{\widetilde{p}\left(X_{i}\right)}\right)+O\left(h^{r}\right), \\
E\left(G\left(X_{i}, Z_{i}, z\right) H\left(X_{j}, Z_{j}, z\right) \widetilde{K}_{i j} K_{z, i} K_{z, j}\right)^{2}=\frac{\|K\|_{2}^{2}}{h^{2 m+n}} E\left(G\left(X_{i}, z, z\right)^{2} H\left(X_{i}, z, z\right)^{2} \frac{p\left(X_{i}, z\right)^{2}}{\widetilde{p}\left(X_{i}\right)}\right)+O\left(h^{-2 m-n+2}\right),
\end{gathered}
$$

and $E\left(E\left(G\left(X_{i}, Z_{i}, z\right) H\left(X_{j}, Z_{j}, z\right) \widetilde{K}_{i j} K_{z, i} K_{z, j} \mid X_{i}, Z_{i}\right)^{2}\right)=O\left(h^{-m+2 r+2}\right)$ if $G\left(x_{i}, z, z\right) \equiv 0$ and $H\left(x_{i}, z, z\right) \equiv 0, O\left(h^{-m+2 r}\right)$ if $H\left(x_{i}, z, z\right) \equiv 0, O\left(h^{-m+2}\right)$ if $G\left(x_{i}, z, z\right) \equiv 0$, and $O\left(h^{-m}\right)$, otherwise; (b) for $i<j<k<l, E\left(\widetilde{K}_{i j} K_{z, i} K_{z, j}\right)^{4}=O\left(h^{-6 m-3 n}\right)$, $E\left(\widetilde{K}_{i j}^{2} \widetilde{K}_{i l}^{2} K_{z, i}^{4} K_{z, j}^{2} K_{z, l}^{2}\right)=O\left(h^{-5 m-2 n}\right)$ and $E\left(\widetilde{K}_{i j} \widetilde{K}_{i k} \widetilde{K}_{l j} \widetilde{K}_{l k} K_{z, i}^{2} K_{z, j}^{2} K_{z, k}^{2} K_{z, l}^{2}\right)=O\left(h^{-4 m}\right)$.

Proof: The results of the lemma are consequences of Proposition B.1 below and the assumptions of the lemma. To show the first result of $(a)$, observe that its left-hand side is

$$
\begin{gathered}
\int\left(\int G\left(x_{i}, z_{i}, z\right) H\left(x_{j}, z_{j}, z\right) p\left(x_{i}, z_{i}\right) p\left(x_{j}, z_{j}\right) \widetilde{K}_{h}\left(x_{i}-x_{j}\right) K_{h}\left(z-z_{j}\right) K_{h}\left(z-z_{i}\right) d x_{j} d z_{j} d z_{i}\right) d x_{i} \\
=\int G\left(x_{i}, z, z\right) H\left(x_{i}, z, z\right) p\left(x_{i}, z\right)^{2} d x_{i}+O\left(h^{r}\right)
\end{gathered}
$$

which is also its right-hand side. The second result of $(a)$ follows from the first one since $\widetilde{K}_{h}(x)^{2}=h^{-n}\|\widetilde{K}\|_{2}^{2} \widetilde{K}_{2, h}(x)$ and $K_{h}(z)^{2}=h^{-m}\|K\|_{2}^{2} K_{2, h}(z)$, where $\widetilde{K}_{2, h}(x)=h^{-n} \widetilde{K}(x / h)^{2} /\|\widetilde{K}\|_{2}^{2}$ and $K_{2, h}(z)=h^{-m} K(z / h)^{2} /\|K\|_{2}^{2}$ are kernel functions of order 2. The third result of $(a)$ can be obtained by observing that its left-hand side is

$$
h^{-m}\|K\|_{2}^{2} \int G\left(x_{i}, z_{i}, z\right) p\left(x_{i}, z_{i}\right) K_{2, h}\left(z-z_{i}\right)\left(\int H\left(x_{j}, z_{j}, z\right) p\left(x_{j}, z_{j}\right) K_{h}\left(x_{i}-x_{j}\right) K_{h}\left(z-z_{j}\right) d x_{j} d z_{j}\right)^{2} d x_{i} d z_{i} .
$$

When $H\left(x_{i}, z, z\right) \equiv 0$, for example, the inner integral squared above is $O\left(h^{2 r}\right)$ and hence the full integral is $O\left(h^{2 r-m}\right) \int G\left(x_{i}, z_{i}, z\right) p\left(x_{i}, z_{i}\right) K_{2, h}\left(z-z_{i}\right)=O\left(h^{2 r-m+2}\right)$ if $G\left(x_{i}, z, z\right) \equiv 0$, and $O\left(h^{2 r-m}\right)$, otherwise. The results of part (b) can be proved in a similar, in fact, much simpler way.

The following localization property of kernel functions can be easily proved by using Taylor expansion and the definition of the order of a kernel function. We omit its prove for shortness sake.

Proposition B.1 Let $K$ be a kernel on $\mathbb{R}^{m}$ of order $r \in \mathbb{N}$. Suppose that a function $g: \mathbb{R}^{m} \rightarrow \mathbb{R}$ is $r$-times continuously differentiable in a neighborhood of $z_{0} \in \mathbb{R}^{m}$. Then, as $h \rightarrow 0$,

$$
\int_{\mathbb{R}^{m}} g(z) K_{h}\left(z-z_{0}\right) d z=g\left(z_{0}\right)+O\left(h^{r}\right) .
$$

Moreover, if the function $g$ has its r-order derivatives bounded on $\mathbb{R}^{m}$, then the term $O\left(h^{r}\right)$ in (B.6) does not depend on $z_{0}$.

The next lemma, implicit in Donald (1997), was used in Section 6 to argue that local rank of a demand system can be estimated from the local rank of a reduced demand system. We omit below dependence on $z$ for shortness sake.

Lemma B.10 Let $f(x)=\left(f_{1}(x), \ldots, f_{J}(x)\right)^{\prime}$ be a $J \times 1$ vector of functions such that $\sum_{j=1}^{J} f_{j}(x)=1$. Then,

$$
\operatorname{rk}\{f\}=\operatorname{rk}\{F ; 1\}+1,
$$

where $F(x)$ is a $(J-1) \times 1$ vector obtained by removing an arbitrary coordinate function $f_{j}(x)$ from the vector $f(x)$ and the rank $\operatorname{rk}\{F ; 1\}$ is defined in Definition 1.1 (omitting $z$ ).

Proof: Suppose without loss of generality that the coordinate function $f_{1}(x)$ is eliminated. Set $L=\operatorname{rk}\{f\}$ and let $F^{(1)}(x)$ denote the vector $f(x)$ with the coordinate function $f_{1}(x)$ eliminated. By Definition 1.1, $f(x)=a h(x)$, where $a=\left(a_{k l}\right)$ is a $J \times L$ matrix and $h(x)=\left(h_{l}(x)\right)$ is a $L \times 1$ vector. Since the $J$ shares add up to 1 , this implies that $1=\left(\sum_{k=1}^{J} a_{k 1}\right) h_{1}(x)+\cdots+\left(\sum_{k=1}^{J} a_{k L}\right) h_{L}(x)$. Suppose, for example, that $\sum_{k=1}^{J} a_{k 1} \neq 0$. Then, we have $h_{1}(x)=\left(\sum_{k=1}^{J} a_{k 1}\right)^{-1}-\left(\sum_{k=1}^{J} a_{k 1}\right)^{-1}\left(\sum_{k=1}^{J} a_{k 2}\right) h_{2}(x)-\ldots-$ $\left(\sum_{k=1}^{J} a_{k 1}\right)^{-1}\left(\sum_{k=1}^{J} a_{k L}\right) h_{L}(x)$. Substituting this expression into $f(x)=a h(x)$, we conclude that $F^{(1)}(x)=c+A H(x)$, where $A$ is a $(J-1) \times(L-1)$ matrix, $H(x)$ is a $(L-1) \times 1$ vector and $c$ is a $(J-1) \times 1$ vector. In view of Definition 1.1, this implies that $\operatorname{rk}\left\{F^{(1)} ; 1\right\} \leq L-1$.

To show the converse, observe that, by using (1.3), the elements $f_{2}(x), \ldots, f_{J}(x)$ of $F^{(1)}(x)$ can be expressed as linear combinations of $\operatorname{rk}\left\{F^{(1)} ; 1\right\}+1$ functions of $x$. Since $f_{1}(x)=1-f_{2}(x)-\ldots-f_{J}(x)$, the function $f_{1}(x)$ can be also expressed as a linear combination of these $\operatorname{rk}\left\{F^{(1)} ; 1\right\}+1$ functions. In view of Definition 1.1 , we obtain that $L=\operatorname{rk}\{f\} \leq \operatorname{rk}\left\{F^{(1)} ; 1\right\}+1$. 


\section{Asymptotics for second order $U$-statistics}

The following useful result concerns the limit behavior of a second order $U$-statistic

$$
U_{N}=\frac{2}{(N-1) N} \sum_{1 \leq i<j \leq N} a_{N}\left(W_{i}, W_{j}\right)
$$

where $W_{i}, i=1, \ldots, N$, are i.i.d. random vectors in $\mathbb{R}^{d}$ and $a_{N}: \mathbb{R}^{d} \times \mathbb{R}^{d} \mapsto \mathbb{R}$ is a symmetric kernel (that is, $\left.a_{N}(x, y)=a_{N}(y, x)\right)$. Although it easily follows from the proof of Lemma 3.1 in Powell, Stock and Stoker (1989), the result is often easier to use and yields stronger results than a direct application of Lemma 3.1 in Powell et al. (1989) itself.

Lemma C.1 Let $U_{N}$ be a second order U-statistic defined by (C.1). Then,

$$
U_{N}=E a_{N}\left(W_{i}, W_{j}\right)+O_{p}\left(\sqrt{\frac{E\left(E\left(a_{N}\left(W_{i}, W_{j}\right) \mid W_{i}\right)^{2}\right)}{N}}+\sqrt{\frac{E a_{N}\left(W_{i}, W_{j}\right)^{2}}{N^{2}}}\right) .
$$

PROOF: Let

$$
\widehat{U}_{N}=E a_{N}\left(W_{i}, W_{j}\right)+\frac{2}{N} \sum_{i=1}^{N}\left(E\left(a_{N}\left(W_{i}, W_{j}\right) \mid W_{i}\right)-E a_{N}\left(W_{i}, W_{j}\right)\right)
$$

be the so-called projection of the $U$-statistic $U_{N}$ (see Serfling (1980) or Powell et al. (1989)). Then, as in the proof of Lemma 3.1 in Powell et al. (1989),

$$
E\left(U_{N}-\widehat{U}_{N}\right)^{2}=\frac{2}{(N-1) N} \sum_{1 \leq i<j \leq N} E b_{N}\left(W_{i}, W_{j}\right)^{2}
$$

where $b_{N}\left(W_{i}, W_{j}\right)=a_{N}\left(W_{i}, W_{j}\right)-E\left(a_{N}\left(W_{i}, W_{j}\right) \mid W_{i}\right)-E\left(a_{N}\left(W_{i}, W_{j}\right) \mid W_{j}\right)+E a_{N}\left(W_{i}, W_{j}\right)$. Since $E b_{N}\left(W_{i}, W_{j}\right)^{2}=$ $O\left(E a_{N}\left(W_{i}, W_{j}\right)^{2}\right)$, we obtain that $E\left(U_{N}-\widehat{U}_{N}\right)^{2}=O\left(E a_{N}\left(W_{i}, W_{j}\right)^{2} / N^{2}\right)$ or

$$
U_{N}-\widehat{U}_{N}=O_{p}\left(\sqrt{\frac{E a_{N}\left(W_{i}, W_{j}\right)^{2}}{N^{2}}}\right)
$$

By the independence of $E\left(a_{N}\left(W_{i}, W_{j}\right) \mid W_{i}\right)$ for different $i$ 's and by using the formula $E(\xi-E \xi)^{2} \leq E \xi^{2}$, we have

$$
E\left(\frac{2}{N} \sum_{i=1}^{N}\left(E\left(a_{N}\left(W_{i}, W_{j}\right) \mid W_{i}\right)-E a_{N}\left(W_{i}, W_{j}\right)\right)\right)^{2}=\frac{4}{N} E\left(E\left(a_{N}\left(W_{i}, W_{j}\right) \mid W_{i}\right)-E a_{N}\left(W_{i}, W_{j}\right)\right)^{2} \leq \frac{4 E\left(E\left(a_{N}\left(W_{i}, W_{j}\right) \mid W_{i}\right)^{2}\right)}{N} .
$$

The result (C.2) then follows from (C.3) and (C.4).

\section{References}

Cragg, J. G. \& Donald, S. G. (1996), On the asymptotic properties of LDU-based tests of the rank of a matrix. Journal of the American Statistical Association 9(435), 1301-1309.

Cragg, J. G. \& Donald, S. G. (1997), Inferring the rank of a matrix. Journal of Econometrics 76(12), 223-250.

de Jong, P. (1987), A central limit theorem for generalized quadratic forms. Probability Theory and Related Fields 75(2), 261-277.

Donald, S. G. (1997), Inference concerning the number of factors in a multivariate nonparametric relationship. Econometrica 65(1), 103-131.

Donald, S. G., Fortuna, N. \& Pipiras, V. (2005), Local and global rank tests for multivariate varyingcoefficient models, FEP Working Paper 196, December, Faculdade de Economia do Porto, Porto, Portugal. 
Fortuna, N. (2004), Local rank tests in a multivariate nonparametric relationship, FEP Working Paper 137, February, Faculdade de Economia do Porto, Porto, Portugal.

Fujikoshi, Y. (1977), Asymptotic expansions for the distributions of some multivariate tests, in Multivariate analysis, IV (Proc. Fourth Internat. Sympos., Dayton, Ohio, 1975), North-Holland, Amsterdam, pp. 55-71.

Gill, L. \& Lewbel, A. (1992), Testing the rank and definiteness of estimated matrices with applications to factor, state-space and ARMA models. Journal of the American Statistical Association 87(419), 766-776.

Gorman, W. M. (1981), Some Engel curves, in A. Deaton, ed., Essays in the theory and measurement of consumer behaviour: in honour of Sir Richard Stone, Cambridge University Press, Cambridge, pp. $7-29$.

Hausman, J. A., Newey, W. K. \& Powell, J. L. (1995), Nonlinear errors in variables: estimation of some Engel curves. Journal of Econometrics 65(1), 205-233.

Kleibergen, F. \& Paap, R. (2006), Generalized reduced rank tests using the singular value decomposition. Journal of Econometrics 133(1), 97-126.

Kneip, A. (1994), Nonparametric estimation of common regressors for similar curve data. The Annals of Statistics 22(3), 1386-1427.

Lewbel, A. (1989), A demand system rank theorem. Econometrica 57(3), 701-705.

Lewbel, A. (1991), The rank of demand systems: theory and nonparametric estimation. Econometrica $59(3), 711-730$.

Lewbel, A. \& Perraudin, W. (1995), A theorem on portfolio separation with general preferences. Journal of Economic Theory 65(2), 624-626.

Magnus, J. R. \& Neudecker, H. (1999), Matrix Differential Calculus with Applications in Statistics and Econometrics, John Wiley \& Sons Ltd., Chichester. Revised reprint of the 1988 original.

Nicol, C. J. (2001), The rank and model specification of demand systems: an empirical analysis using United States microdata. Canadian Journal of Economics 34(1), 259-289.

Pagan, A. \& Ullah, A. (1999), Nonparametric Econometrics, Cambridge University Press, Cambridge.

Pötscher, B. M. (1983), Order estimation in ARMA-models by Lagrangian multiplier tests. The Annals of Statistics 11(3), 872-885.

Powell, J. L., Stock, J. H. \& Stoker, T. M. (1989), Semiparametric estimation of index coefficients. Econometrica 57(6), 1403-1430.

Rao, C. R. (1973), Linear Statistical Inference and its Applications, Second edn, John Wiley \& Sons, New York-London-Sydney. Wiley Series in Probability and Mathematical Statistics.

Robin, J.-M. \& Smith, R. J. (2000), Tests of rank. Econometric Theory 16(2), 151-175.

Russell, T. \& Farris, F. (1993), The geometric structure of some systems of demand equations. Journal of Mathematical Economics 22(4), 309-325. 
Serfling, R. J. (1980), Approximation Theorems of Mathematical Statistics, John Wiley \& Sons Inc., New York. Wiley Series in Probability and Mathematical Statistics.

Silverman, B. W. (1986), Density Estimation for Statistics and Data Analysis, Chapman and Hall, New York.

White, H. \& Hong, Y. (1999), M-testing using finite and infinite dimensional parameter estimators, Discussion paper 93-01R, Department of Economics, University of California, San Diego. 


\begin{tabular}{|c|c|c|c|c|c|c|c|c|}
\hline \multicolumn{3}{|c|}{ Local rank } & \multicolumn{3}{|c|}{$1(z=-1 / 2)$} & \multicolumn{3}{|c|}{$2(z=0)$} \\
\hline$N$ & $\delta$ & $h_{x} \backslash h_{z}$ & 0.1 & 0.3 & 0.5 & 0.1 & 0.3 & 0.5 \\
\hline \multirow{8}{*}{750} & \multirow{4}{*}{1} & 0.1 & 6.1 & 5.1 & 9.6 & 0.1 & 1.0 & 2.3 \\
\hline & & 0.3 & 3.3 & 4.7 & 14.4 & 0.1 & 0.5 & 1.5 \\
\hline & & 0.5 & 2.4 & 4.1 & 16.8 & 0.1 & 0.5 & 1.9 \\
\hline & & $\min -\chi^{2}$ & 7.0 & 8.3 & 24.6 & 2.0 & 2.7 & 6.4 \\
\hline & \multirow{4}{*}{$1 / 2$} & 0.1 & 4.8 & 3.6 & 6.4 & 0.1 & 0.4 & 0.2 \\
\hline & & 0.3 & 2.5 & 3.7 & 5.4 & 0.0 & 0. & 0.3 \\
\hline & & 0.5 & 1.9 & 2.3 & 5.7 & 0.0 & 0.2 & 1.2 \\
\hline & & $\min -\chi^{2}$ & 6.9 & 6.4 & 9.1 & 0.5 & 0.8 & 1.7 \\
\hline \multirow{8}{*}{1500} & \multirow{4}{*}{1} & 0.1 & 3.1 & 3.5 & 18.3 & 0.8 & 1.0 & 3.3 \\
\hline & & 0.3 & 2.0 & 2.6 & 30.6 & 0.3 & 0.6 & 5.0 \\
\hline & & 0.5 & 1.6 & 2.7 & 36.8 & 0.3 & 0.5 & 6.5 \\
\hline & & $\min -\chi^{2}$ & 5.3 & 5.1 & 45.0 & 3.2 & 5.2 & 14.5 \\
\hline & \multirow{4}{*}{$1 / 2$} & 0.1 & 2.9 & 2.6 & 6.9 & 0.2 & 0.3 & 0.5 \\
\hline & & 0.3 & 2.1 & 1.9 & 7.3 & 0.2 & 0.1 & 0.5 \\
\hline & & 0.5 & 1.6 & 1.5 & 9.4 & 0.1 & 0.0 & 0.7 \\
\hline & & $\min -\chi^{2}$ & 5.2 & 3.8 & 13.3 & 1.3 & 1.7 & 4.9 \\
\hline
\end{tabular}

Table 1: Size of local rank tests using $\widehat{T}_{1}$ 


\begin{tabular}{|c|c|c|c|c|c|c|c|c|c|c|c|}
\hline \multirow{2}{*}{\multicolumn{3}{|c|}{$\begin{array}{l}\text { True local rank } \\
\text { Local rank test }\end{array}$}} & \multirow{2}{*}{\multicolumn{3}{|c|}{$\begin{array}{c}L_{0}=2 \\
L=1\end{array}$}} & \multicolumn{6}{|c|}{$L_{0}=3$} \\
\hline & & & & & & & $L=1$ & & & $L=2$ & \\
\hline$N$ & $\delta$ & $h_{x} \backslash h_{z}$ & 0.1 & 0.3 & 0.5 & 0.1 & 0.3 & 0.5 & 0.1 & 0.3 & 0.5 \\
\hline \multirow{8}{*}{750} & \multirow{4}{*}{1} & 0.1 & 17.8 & 42.4 & 52.4 & 89.7 & 100.0 & 100.0 & 51.7 & 93.3 & 98.7 \\
\hline & & 0.3 & 23.4 & 55.9 & 58.3 & 97.1 & 100.0 & 100.0 & 67.7 & 98.5 & 99.9 \\
\hline & & 0.5 & 25.6 & 61.7 & 49.6 & 99.1 & 100.0 & 100.0 & 74.4 & 98.8 & 99.7 \\
\hline & & $\min -\chi^{2}$ & 23.4 & 59.9 & 56.5 & 97.7 & 100.0 & 100.0 & 80.1 & 99.4 & 99.7 \\
\hline & \multirow{4}{*}{$1 / 2$} & 0.1 & 6.9 & 13.7 & 12.1 & 27.8 & 85.1 & 99.0 & 18.2 & 42.4 & 63.8 \\
\hline & & 0.3 & 9.4 & 14.5 & 17.7 & 46.9 & 96.2 & 100.0 & 26.5 & 57.5 & 72.6 \\
\hline & & 0.5 & 7.0 & 16.8 & 15.7 & 49.2 & 97.6 & 100.0 & 28.9 & 63.9 & 75.7 \\
\hline & & $\min -\chi^{2}$ & 7.6 & 17.9 & 20.7 & 47.3 & 97.1 & 100.0 & 29.8 & 69.6 & 82.6 \\
\hline \multirow{8}{*}{1500} & \multirow{4}{*}{1} & 0.1 & 33.7 & 81.8 & 83.2 & 99.9 & 100.0 & 100.0 & 78.8 & 99.7 & 100.0 \\
\hline & & 0.3 & 50.9 & 94.0 & 88.6 & 100.0 & 100.0 & 100.0 & 92.3 & 100.0 & 100.0 \\
\hline & & 0.5 & 52.8 & 94.4 & 81.8 & 100.0 & 100.0 & 100.0 & 93.6 & 100.0 & 100.0 \\
\hline & & $\min -\chi^{2}$ & 49.6 & 94.3 & 83.7 & 100.0 & 100.0 & 100.0 & 96.0 & 100.0 & 100.0 \\
\hline & \multirow{4}{*}{$1 / 2$} & 0.1 & 11.1 & 22.4 & 24.0 & 64.5 & 99.8 & 100.0 & 31.2 & 73.1 & 87.9 \\
\hline & & 0.3 & 16.3 & 35.5 & 33.8 & 84.7 & 100.0 & 100.0 & 41.4 & 85.9 & 95.1 \\
\hline & & 0.5 & 16.1 & 38.1 & 30.6 & 88.4 & 100.0 & 100.0 & 45.6 & 88.3 & 95.4 \\
\hline & & $\min -\chi^{2}$ & 16.0 & 39.2 & 35.0 & 86.0 & 100.0 & 100.0 & 51.2 & 90.3 & 96.4 \\
\hline
\end{tabular}

Table 2: Power of local rank tests using $\widehat{T}_{1}$ 


\begin{tabular}{|c|c|c|c|c|c|c|c|c|c|c|c|}
\hline \multicolumn{3}{|c|}{ True rank } & \multicolumn{3}{|c|}{$L_{0}=1$} & \multicolumn{3}{|c|}{$L_{0}=2$} & \multicolumn{3}{|c|}{$L_{0}=3$} \\
\hline$\delta$ & $h_{x}$ & $h_{z}$ & $\widehat{L}=1$ & $\widehat{L}=2$ & $\widehat{L}=3$ & $\widehat{L}=1$ & $\widehat{L}=2$ & $\widehat{L}=3$ & $\widehat{L}=1$ & $\widehat{L}=2$ & $\widehat{L}=3$ \\
\hline \multirow{12}{*}{1} & \multirow{3}{*}{0.1} & 0.1 & 0.939 & 0.060 & 0.001 & 0.809 & 0.190 & 0.001 & 0.095 & 0.647 & 0.258 \\
\hline & & 0.3 & 0.949 & 0.051 & 0.000 & 0.571 & 0.419 & 0.010 & 0.000 & 0.143 & 0.857 \\
\hline & & 0.5 & 0.904 & 0.093 & 0.003 & 0.351 & 0.626 & 0.023 & 0.000 & 0.030 & 0.970 \\
\hline & \multirow{3}{*}{0.3} & 0.1 & 0.967 & 0.033 & 0.000 & 0.810 & 0.189 & 0.001 & 0.041 & 0.622 & 0.337 \\
\hline & & 0.3 & 0.953 & 0.046 & 0.001 & 0.457 & 0.538 & 0.005 & 0.000 & 0.079 & 0.921 \\
\hline & & 0.5 & 0.856 & 0.140 & 0.004 & 0.222 & 0.763 & 0.015 & 0.000 & 0.006 & 0.994 \\
\hline & \multirow{3}{*}{0.5} & 0.1 & 0.976 & 0.024 & 0.000 & 0.806 & 0.193 & 0.001 & 0.020 & 0.659 & 0.321 \\
\hline & & 0.3 & 0.959 & 0.041 & 0.000 & 0.432 & 0.563 & 0.005 & 0.000 & 0.070 & 0.930 \\
\hline & & 0.5 & 0.832 & 0.164 & 0.004 & 0.208 & 0.773 & 0.019 & 0.000 & 0.004 & 0.996 \\
\hline & \multirow{3}{*}{$\min -\chi^{2}$} & 0.1 & 0.930 & 0.065 & 0.005 & 0.694 & 0.286 & 0.020 & 0.016 & 0.280 & 0.704 \\
\hline & & 0.3 & 0.917 & 0.078 & 0.005 & 0.331 & 0.642 & 0.027 & 0.000 & 0.015 & 0.985 \\
\hline & & 0.5 & 0.754 & 0.227 & 0.019 & 0.162 & 0.774 & 0.064 & 0.000 & 0.002 & 0.998 \\
\hline \multirow{12}{*}{$1 / 2$} & \multirow{3}{*}{0.1} & 0.1 & $\begin{array}{l}0.898 \\
\end{array}$ & 0.047 & 0.001 & 0.911 & 0.068 & 0.001 & 0.718 & 0.263 & 0.015 \\
\hline & & 0.3 & 0.964 & 0.036 & 0.000 & 0.898 & 0.098 & 0.004 & 0.174 & 0.698 & 0.128 \\
\hline & & 0.5 & 0.936 & 0.064 & 0.000 & 0.859 & 0.139 & 0.002 & 0.007 & 0.683 & 0.310 \\
\hline & \multirow{3}{*}{0.3} & 0.1 & 0.953 & 0.025 & 0.000 & 0.922 & 0.061 & 0.000 & 0.633 & 0.347 & 0.018 \\
\hline & & 0.3 & 0.963 & 0.037 & 0.000 & 0.889 & 0.110 & 0.001 & 0.054 & 0.726 & 0.220 \\
\hline & & 0.5 & 0.946 & 0.053 & 0.001 & 0.810 & 0.187 & 0.003 & 0.000 & 0.580 & 0.420 \\
\hline & \multirow{3}{*}{0.5} & 0.1 & 0.957 & 0.019 & 0.000 & 0.942 & 0.053 & 0.000 & 0.613 & 0.377 & 0.010 \\
\hline & & 0.3 & 0.977 & 0.023 & 0.000 & 0.872 & 0.126 & 0.002 & 0.037 & 0.751 & 0.212 \\
\hline & & 0.5 & 0.943 & 0.055 & 0.002 & 0.830 & 0.168 & 0.002 & 0.000 & 0.593 & 0.407 \\
\hline & \multirow{3}{*}{$\min -\chi^{2}$} & 0.1 & 0.911 & 0.064 & 0.005 & 0.889 & 0.103 & 0.005 & 0.464 & 0.409 & 0.127 \\
\hline & & 0.3 & 0.936 & 0.060 & 0.004 & 0.796 & 0.196 & 0.008 & 0.023 & 0.471 & 0.506 \\
\hline & & 0.5 & 0.909 & 0.085 & 0.006 & 0.717 & 0.266 & 0.017 & 0.000 & 0.289 & 0.711 \\
\hline
\end{tabular}

Table 3: Empirical distribution of $\widehat{L}$ using $\widehat{T}_{1}(N=750, \alpha=0.05)$ 


\begin{tabular}{cccccc}
\hline \multirow{2}{*}{$N$} & $\delta$ & $h_{x}$ & $\widehat{L}=1$ & $\widehat{L}=2$ & $\widehat{L}=3$ \\
\hline \multirow{4}{*}{750} & & 0.1 & 0.000 & 0.189 & 0.811 \\
& & 0.3 & 0.000 & 0.103 & 0.897 \\
& & 0.5 & 0.000 & 0.106 & 0.894 \\
\cline { 2 - 5 } & & min- $\chi^{2}$ & 0.000 & 0.047 & 0.953 \\
\cline { 2 - 5 } & \multirow{4}{*}{$1 / 2$} & 0.1 & 0.007 & 0.825 & 0.168 \\
& & 0.3 & 0.001 & 0.777 & 0.222 \\
& & 0.5 & 0.001 & 0.788 & 0.211 \\
\cline { 3 - 6 } & & min- $\chi^{2}$ & 0.000 & 0.662 & 0.337 \\
\hline
\end{tabular}

Table 4: Empirical distribution of $\widehat{L}$ ignoring $z(\alpha=0.05)$ 


\begin{tabular}{|c|c|c|c|c|c|c|c|c|c|c|c|}
\hline \multicolumn{3}{|c|}{ Local rank test } & \multicolumn{3}{|c|}{$L=1$} & \multicolumn{3}{|c|}{$L=2$} & \multicolumn{3}{|c|}{$L=3$} \\
\hline$z$ & Statistic & $h_{x} \backslash h_{z}$ & 0.05 & 0.09 & 0.15 & 0.05 & 0.09 & 0.15 & 0.05 & 0.09 & 0.15 \\
\hline \multirow{6}{*}{1} & \multirow{3}{*}{$\widehat{T}_{1}$} & 0.1 & 0.000 & 0.000 & 0.000 & 0.327 & 0.001 & 0.000 & 0.948 & 0.957 & 0.885 \\
\hline & & 0.3 & 0.000 & 0.000 & 0.000 & 0.067 & 0.000 & 0.000 & 0.955 & 0.953 & 0.896 \\
\hline & & 0.55 & 0.000 & 0.000 & 0.000 & 0.051 & 0.000 & 0.000 & 0.980 & 0.986 & 0.971 \\
\hline & \multirow{3}{*}{$\widehat{T}_{2}$} & 0.1 & 0.000 & 0.000 & 0.000 & 0.055 & 0.000 & 0.000 & 0.653 & 0.493 & 0.763 \\
\hline & & 0.3 & 0.000 & 0.000 & 0.000 & 0.000 & 0.000 & 0.000 & 0.689 & 0.770 & 0.791 \\
\hline & & 0.55 & 0.000 & 0.000 & 0.000 & 0.000 & 0.000 & 0.000 & 0.614 & 0.407 & 0.333 \\
\hline \multirow{6}{*}{1.2} & \multirow{3}{*}{$\widehat{T}_{1}$} & 0.1 & 0.242 & 0.040 & 0.026 & 0.556 & 0.276 & 0.250 & 0.752 & 0.586 & 0.599 \\
\hline & & 0.3 & 0.213 & 0.068 & 0.030 & 0.505 & 0.591 & 0.355 & 0.767 & 0.913 & 0.918 \\
\hline & & 0.55 & 0.152 & 0.018 & 0.006 & 0.402 & 0.459 & 0.282 & 0.711 & 0.891 & 0.928 \\
\hline & \multirow{3}{*}{$\widehat{T}_{2}$} & 0.1 & 0.991 & 0.566 & 0.588 & 0.998 & 0.808 & 0.938 & 0.989 & 0.725 & 0.985 \\
\hline & & 0.3 & 0.982 & 0.137 & 0.059 & 0.990 & 0.764 & 0.257 & 0.984 & 0.809 & 0.871 \\
\hline & & 0.55 & 0.975 & 0.022 & 0.001 & 0.982 & 0.637 & 0.098 & 0.990 & 0.872 & 0.870 \\
\hline
\end{tabular}

Table 5: $P$-values in rank estimation at $z=1$ and $z=1.2$ 